\title{
ROTATIONAL VELOCITIES OF INDIVIDUAL COMPONENTS IN VERY LOW MASS BINARIES
}

\author{
Q. M. Konopacky ${ }^{1,10,11}$, A. M. GheZ ${ }^{2}$, D. C. FabrycKY ${ }^{3,12}$, B. A. Macintosh ${ }^{1}$, R. J. White ${ }^{4}$, \\ T. S. Barman ${ }^{5}$, E. L. Rice ${ }^{6,13}$, G. Hallinan ${ }^{7}$, AND G. DuchêNE ${ }^{8,9}$ \\ ${ }^{1}$ Lawrence Livermore National Laboratory, 7000 East Avenue, Livermore, CA 94550, USA; macintosh1@1lnl.gov, konopacky@di.utoronto.ca \\ ${ }^{2}$ UCLA Division of Astronomy and Astrophysics, Los Angeles, CA 90095-1562, USA; ghez@astro.ucla.edu \\ ${ }^{3}$ Department of Astronomy and Astrophysics, University of California, Santa Cruz, CA 95064, USA; fabrycky@ucolick.org \\ ${ }^{4}$ Department of Physics and Astronomy, Georgia State University, Atlanta, GA 30303, USA; white@ chara.gsu.edu \\ ${ }^{5}$ Lowell Observatory, 1400 W. Mars Hill Rd., Flagstaff, AZ 86001, USA; barman@lowell.edu \\ ${ }^{6}$ American Museum of Natural History, Central Park West at 79th Street, New York, NY 10024-5192, USA; erice@ amnh.org \\ ${ }^{7}$ Department of Astrophysics, California Institute of Technology, MC 249-17, Pasadena, CA 91125, USA; gh@ astro.caltech.edu \\ ${ }^{8}$ Astronomy Department, University of California, Berkeley, Hearst Field Annex B-20, CA 94720-3411, USA; gduchene@ berkeley.edu \\ ${ }^{9}$ UJF-Grenoble 1/CNRS-INSU, Institut de Planétologie et d'Astrophysique de Grenoble (IPAG) UMR 5274, Grenoble F-38041, France \\ Received 2011 July 8; accepted 2012 February 24; published 2012 April 17
}

\begin{abstract}
We present rotational velocities for individual components of 11 very low mass (VLM) binaries with spectral types between M7 and L7.5. These results are based on observations taken with the near-infrared spectrograph, NIRSPEC, and the Keck II laser guide star adaptive optics system. We find that the observed sources tend to be rapid rotators $\left(v \sin i>10 \mathrm{~km} \mathrm{~s}^{-1}\right.$ ), consistent with previous seeing-limited measurements of VLM objects. The two sources with the largest $v \sin i$, LP 349-25B and HD 130948C, are rotating at $\sim 30 \%$ of their break-up speed, and are among the most rapidly rotating VLM objects known. Furthermore, five binary systems, all with orbital semimajor axes $\lesssim 3.5 \mathrm{AU}$, have component $v$ sin $i$ values that differ by greater than $3 \sigma$. To bring the binary components with discrepant rotational velocities into agreement would require the rotational axes to be inclined with respect to each other, and that at least one component is inclined with respect to the orbital plane. Alternatively, each component could be rotating at a different rate, even though they have similar spectral types. Both differing rotational velocities and inclinations have implications for binary star formation and evolution. We also investigate possible dynamical evolution in the triple system HD 130948A-BC. The close binary brown dwarfs B and C have significantly different $v$ sin $i$ values. We demonstrate that components $\mathrm{B}$ and $\mathrm{C}$ could have been torqued into misalignment by the primary star, A, via orbital precession. Such a scenario can also be applied to another triple system in our sample, GJ 569A-Bab. Interactions such as these may play an important role in the dynamical evolution of VLM binaries. Finally, we note that two of the binaries with large differences in component $v \sin i$, LP 349-25AB and 2MASS $0746+20 \mathrm{AB}$, are also known radio sources.
\end{abstract}

Key words: binaries: visual - brown dwarfs - stars: fundamental parameters - stars: low-mass - stars: rotation

Online-only material: color figures

\section{INTRODUCTION}

Rotational velocity is an important diagnostic parameter for stellar objects, offering a window into the angular momentum evolution of a given source. A star's rotation can provide important clues to its formation and can furnish diagnostics of its interior structure and evolution. For instance, measurements of rotational velocity have been shown to correlate strongly with stellar activity, possibly driving the magnetic dynamo responsible for generating this activity (Browning 2008). In addition, rotational velocities have been shown to correlate with the age of a system, offering a tool for estimating stellar ages (Delfosse et al. 1998).

The rotational behavior of very low mass (VLM) stars and brown dwarfs has been studied by a number of authors in recent years (Mohanty \& Basri 2003; Bailer-Jones 2004; Zapatero Osorio et al. 2006; Reiners \& Basri 2008, 2010; Blake et al.

\footnotetext{
${ }^{10}$ Current address: Dunlap Institute for Astronomy and Astrophysics, University of Toronto, 50 St. George Street, Toronto M5S 3H4, Ontario, Canada.

${ }^{11}$ Hubble Fellow.

12 Dunlap Fellow.

${ }^{13}$ Current address: College of Staten Island, City University of New York, 2800 Victory Blvd., Staten Island, NY 10314, USA.
}

2010). It has been shown that the brown dwarfs tend to be rapid rotators, and that the minimum rotation rate is a function of spectral type (i.e., Zapatero Osorio et al. 2006; Reiners \& Basri 2008). It has also been determined that the rotational velocities of brown dwarfs correlate with age, with VLM objects having very long spin-down timescales, and that their rotational evolution is probably dominated primarily by magnetic braking (e.g., Reiners \& Basri 2008; Scholz et al. 2009, 2011). However, it appears that the activity-rotation relationship that is very strong among $\mathrm{M}$ dwarfs tends to break down at these low masses (Mohanty \& Basri 2003). In spite of this, activity in the form of radio emission has been observed in a number of VLM systems (e.g., Berger 2006; Osten et al. 2006; Hallinan et al. 2008). In addition, it has been shown that this drop in activity does not seem to be due to a reduction in magnetic field strengths in late M dwarfs, but might instead be due to their reduced temperature and hence reduced fractional ionization of their atmospheres (e.g., Reiners \& Basri 2007; Hallinan et al. 2006, 2008).

The majority of previous studies have been performed with seeing-limited observations, and most sources targeted are thought to be single. Known binaries have been included in various samples, and their rotational velocities have been derived from the combined light of both components. The rotational velocities of individual binary components can potentially provide 
a unique look at the rotational evolution of VLM objects. If any differences are seen between the velocities of the binary components, it could have implications for the way in which these binaries formed, their early accretion history, or the operation of magnetic braking as a function of mass. For instance, Reiners et al. (2007) found that the components of the triple system LHS 1070 (spectral types M5.5, M9, and M9) had differing $v \sin i$, with the higher mass component rotating about a factor of two more slowly than the two lower mass objects. This allowed the authors to put constraints on the form of rotational braking in the VLM regime. Further, Gómez Maqueo Chew et al. (2009) found that the components of the young eclipsing binary brown dwarf 2MASS 0535-05AB (spectral types M6.5) have different rotational periods, with the primary component rotating more rapidly than the secondary.

If the orbits of these binaries are known, rotational velocities provide a way to test the assumption that spin axes are generally perpendicular to the orbital plane. Work by Hale (1994) found that, in general, binaries with separations $\lesssim 30-40$ AU should have spin axes perpendicular to their orbital plane. However, some very close (semimajor axis $\lesssim 0.3 \mathrm{AU}$ ) binaries such as DI Herculis (Albrecht et al. 2009) have been shown to have extremely misaligned axes. In contrast, more recent work on a very similar binary system (NY Cep; Albrecht et al. 2011) has revealed no such misalignment, implying that the cause of the inclined axes is non-universal. It is also important to explore systems with wider separations that may not be subject to the same extreme tidal interactions as very close binaries. This has been done in the case of some T Tauri binary systems, which have shown both slight and substantial planar misalignment via observations of disk orientation (Jensen et al. 2004; Monin et al. 2006). Probing the rotational evolution of intermediate separation binaries $(\sim 1-10 \mathrm{AU})$ in the substellar regime will determine whether such trends hold at the lowest masses, with interesting implications for the formation and evolution of all types of binary stars.

In this paper, we present projected rotational velocity measurements for the components of a sample of tight, visual VLM binaries. The measurements of these spatially resolved velocities are enabled by the W.M. Keck Observatory laser guide star adaptive optics (LGS AO) system, which provides high spatial resolution observations of optically faint targets (Wizinowich et al. 2006). This study is the first to systematically examine the rotational velocities of individual VLM objects that reside in binary systems. In Section 2, we describe our observations and our method for extracting rotational velocities from highresolution spectra. In Section 3, we compare our measurements to those of single VLM objects, and discuss the implications of our measurements for theories of binary star formation and evolution. We also discuss our results in the context of previously measured radio emission from two of our sources. We summarize our findings in Section 4.

\section{DATA AND ANALYSIS}

\subsection{Sample}

Our sample is comprised of 11 VLM binaries that were targeted as part of an ongoing program to measure their dynamical masses. These objects have been observed both astrometrically and spectroscopically since 2006, and initial estimates of their orbital properties have been obtained from this data set (Konopacky et al. 2010). Their spectral types range from M7.5 to L7.5, and their separations range from 0 '.07 to 0 '.35.
Because we are able to spatially resolve the components before obtaining high-resolution spectroscopy (see Section 2.2), our total sample consists of 22 VLM objects. Table 1 summarizes the targets in our sample.

\subsection{Observations}

The 11 binaries were observed using the NIR spectrograph NIRSPEC on Keck II 10 m (McLean et al. 2000) in conjunction with the facility LGS AO system (NIRSPAO). These observations, taken between 2006 December and 2011 June, are described in detail in Konopacky et al. (2010). Briefly, we used the instrument in its high spectral resolution mode, selecting a slit 0.041 in width and 2".26 in length in AO mode. We observed in the $K$ band in order to obtain data in the $\mathrm{CO}$ band head region (2.291-2.325 $\mu \mathrm{m}$, order 33). Due to the dense population of lines in this region, our analysis for this work was done only in order 33, although the cross-dispersed data ranged from 2.044 to $2.382 \mu \mathrm{m}$.

The camera was rotated such that both components of each binary fell simultaneously on the high-resolution slit, which is at an angle of 105.9 with respect to vertical. Typical observations consisted of four spectra of both components, each with 900-1800 s integration times, taken in an ABBA dither pattern along the length of the slit. On average, we achieved Strehl ratios between $10 \%$ and $40 \%$ at the $K$ band, resulting in point-spread function core FWHMs of $\sim 0$ '.05-0'08. As discussed in Konopacky et al. (2010), this performance allowed us, in general, to obtain resolved spectra for binaries separated by at least $\sim 0$.'06.

Table 2 gives the log of our spectroscopic observations, listing the targets observed, the date of observation, the number of spectra, the integration time for each spectrum, and the average signal-to-noise ratio $(\mathrm{S} / \mathrm{N})$ achieved. Because the spectra are dominated by molecular features (see Figure 2), we have chosen to estimate effective $\mathrm{S} / \mathrm{N}$ by calculating the average number of electrons per pixel in an extracted spectrum and then assuming Poissonian noise on that average. We have verified that these estimates are roughly correct by calculating the $\mathrm{S} / \mathrm{N}$ on small regions of our most rapidly rotating source, where all features are fairly smoothed out, and by using the properties of the NIRSPEC detector under the assumption that we are background limited at the $K$ band. ${ }^{14}$ Each target observation was accompanied by the observation of a nearby $\mathrm{A} 0 \mathrm{~V}$ star to measure the telluric absorption.

\subsection{Data Reduction}

As described in Konopacky et al. (2010), the basic reduction of the NIRSPAO spectra was performed with REDSPEC, a software package designed for NIRSPEC. ${ }^{15}$ Object frames are reduced by subtracting opposing nods to remove sky and dark backgrounds, dividing by a flat field, and correcting for bad pixels. Order 33 was spatially rectified by fitting the trace of each nod of A0 calibrators with third-order polynomials, and then applying the results of those fits across the image. As these systems are fairly tight binaries, cross-contamination can be an issue when extracting the spectra. We use a Gaussian extraction method to extract the spectra, fitting the trace with a variable FWHM in cases where the separation was greater than 7 pixels and a fixed FWHM if less than 7 pixels. We subtract the results

\footnotetext{
14 http://www2.keck.hawaii.edu/inst/nirspec/sens.html

15 http://www2.keck.hawaii.edu/inst/nirspec/redspec.html
} 
Table 1

VLM Binary Sample

\begin{tabular}{|c|c|c|c|c|c|}
\hline Source Name & $\begin{array}{l}\text { R.A. } \\
\text { (J2000) }\end{array}$ & $\begin{array}{l}\text { Decl. } \\
\text { (J2000) }\end{array}$ & $\begin{array}{l}\text { Estimated } \\
\text { Sp. Types }\end{array}$ & $\begin{array}{l}\text { Discovery } \\
\text { Reference }\end{array}$ & $\begin{array}{c}\text { 2MASS } \\
K \text {-band Mag. }\end{array}$ \\
\hline LP $349-25 \mathrm{AB}$ & 002755.93 & +221932.8 & M8+M9 & 1 & $9.569 \pm 0.017$ \\
\hline LP $415-20 A B$ & 042149.0 & +192910 & M7+M9.5 & 2 & $11.668 \pm 0.020$ \\
\hline 2MASS J07464256+2000321AB & 074642.5 & +200032 & $\mathrm{~L} 0+\mathrm{L} 1.5$ & 3 & $10.468 \pm 0.022$ \\
\hline GJ 569Bab & 145429.0 & +160605 & M8.5+M9 & 4 & $\sim 9.8$ \\
\hline LHS 2397aAB & 112149.25 & -131308.4 & M8+L7.5 & 5 & $10.735 \pm 0.023$ \\
\hline 2MASS J14263161+1557012AB & 142631.62 & +15 5701.3 & $\mathrm{M} 8.5+\mathrm{L} 1$ & 6 & $11.731 \pm 0.018$ \\
\hline HD 130948BC & 145015.81 & +235442.6 & L4+L4 & 7 & $\sim 11.0$ \\
\hline 2MASS J17501291+4424043AB & 175012.91 & +442404.3 & $\mathrm{M} 7.5+\mathrm{L} 0$ & 2 & $11.768 \pm 0.017$ \\
\hline 2MASS J18470342+5522433AB & 184703.42 & +552243.3 & $\mathrm{M} 7+\mathrm{M} 7.5$ & 8 & $10.901 \pm 0.020$ \\
\hline 2MASS J21402931+1625183AB & 214029.32 & +162518.3 & $\mathrm{M} 8.5+\mathrm{L} 2$ & 6 & $11.826 \pm 0.031$ \\
\hline 2MASS J22062280-2047058AB & 220622.80 & -204705.9 & M8+M8 & 6 & $11.315 \pm 0.027$ \\
\hline
\end{tabular}

Notes.

${ }^{a}$ From discovery reference.

References. (1) Forveille et al. 2005; (2) Siegler et al. 2003; (3) Reid et al. 2001; (4) Martín et al. 2000; (5) Freed et al. 2003; (6) Close et al. 2003; (7) Potter et al. 2002; (8) Siegler et al. 2005.

of this fit for one component before extracting the other (see Konopacky et al. 2010 for more details).

\subsection{Determination of $v \sin i$}

The data set presented here is the same set presented in Konopacky et al. (2010), except we now include three new epochs of data from 2009 December, 2010 June, and 2011 June. In Konopacky et al. (2010), we were primarily interested in the radial velocities, and hence orbital solutions, that could be derived from these spectra. Here, we reanalyze these data having implemented two changes to our analysis in order to properly determine $v \sin i$ (Bailey et al. 2012). First, we now use the telluric lines present in the spectra of the A0V calibrator stars to measure the instrumental profile for NIRSPAO. Second, we are now performing the convolution of theoretical templates with a Gaussian kernel after putting all spectra on a loglinear scale. This makes the resolution constant across the entire spectral range, providing a more accurate measure of $v \sin i$. We describe our analysis in more detail below. Note that this reanalysis does not substantially impact our radial velocity estimates, which will be presented in a future paper.

It has been demonstrated that the $\mathrm{CO}$ band head line depths and widths are primarily a function of temperature and the projected rotational velocity ( $v \sin i$ ) for VLM objects, respectively, with an additional moderate dependence on surface gravity (Blake et al. 2007) and metallicity. With some knowledge of the temperature of a given object and an allowance for unknown surface gravity and metallicity, $v$ sin $i$ measurements can be estimated from our extracted spectra.

Our extracted spectra are not corrected for telluric absorption because these features provide a stable reference for absolute wavelength calibration. Using features that are naturally present in all spectra also allows us to accurately calibrate the instrumental profile without the need to observe additional template sources. We therefore model each spectrum as a combination of a KPNO/Fourier transform spectroscope telluric spectrum (Livingston \& Wallace 1991) and a synthetically generated spectrum derived from the PHOENIX atmosphere models (Hauschildt et al. 1999). The model spectrum is parameterized to account for the wavelength solution, continuum normalization, instrumental profile (assumed to be Gaussian), $v$ sin $i$, and radial velocity. As mentioned above, the instrumental profile is determined using our A0V calibrator stars, which by design are a clean measure of the actual telluric spectrum. We hold the instrumental profile fixed while fitting our actual target spectra. The average resolution of our NIRSPAO data is $\sim 10 \mathrm{~km} \mathrm{~s}^{-1}$. The best-fit model is determined by minimizing the varianceweighted reduced $\chi^{2}$ of the difference between the model and the extracted spectrum, once this difference has been Fourier filtered to remove the fringing present in NIRSPEC $K$-band spectra (see Bailey et al. 2012 for more details). This model therefore provides our $v \sin i$ estimates.

Each PHOENIX template is generated at a fixed temperature and surface gravity. The use of theoretical rather than observed templates has the advantage of introducing less template mismatch biases. Our main templates for each source have a temperature as measured in Konopacky et al. (2010), a $\log (g)$ of 5.0 , and solar metallicity. Figure 1 shows example fits for two objects in our sample, one moderate rotator (GJ 569Ba) and one rapid rotator (LP 349-25A) that have different temperatures. The figure demonstrates the location and morphology of the telluric features that are used for estimating the instrumental profile and wavelength solution. Figure 4 of Konopacky et al. (2010) shows example fits for all objects in our sample-we refer the reader to this work for further visual evaluation of our fitting technique.

Statistical uncertainties are assigned by fitting each individual spectrum separately and taking the rms of the values derived for each case. We also need to account for systematic uncertainties due to both the temperature and surface gravity dependence of our spectra. We fit each spectrum with templates spanning $\pm 300 \mathrm{~K}$ in temperature and ranging from 3.0 to $4.5 \mathrm{dex}$ in $\log (g)$. We also explored varying metallicity, using templates between \pm 0.25 dex of solar to fit our spectra based on metallicity measurements of low-mass objects in the solar neighborhood (e.g., Johnson \& Apps 2009; Schlaufman \& Laughlin 2010; Rojas-Ayala et al. 2010). We then use the spread in these values around our best-fit value as our systematic uncertainty, and add these in quadrature with our statistical uncertainties. We find on average that $\log (g)$ uncertainties add a $3 \mathrm{~km} \mathrm{~s}^{-1}$ uncertainty to the $v \sin i$, while temperature and metallicity uncertainties contribute an additional $1 \mathrm{~km} \mathrm{~s}^{-1}$ each, with lower values of temperature and metallicity yielding lower $v \sin i$. The $v \sin i$ 
Table 2

$\log$ of NIRSPAO LGS $K$-band Observations ${ }^{\mathrm{a}}$

\begin{tabular}{|c|c|c|c|c|c|c|}
\hline Target Name & $\begin{array}{c}\text { Date of } \\
\text { Observation (UT) }\end{array}$ & $\begin{array}{l}\text { A0V Star } \\
\text { Standard }\end{array}$ & $\begin{array}{c}\text { Exposure Time } \\
\text { (s) }\end{array}$ & $\begin{array}{l}\text { No. of } \\
\text { Frames }\end{array}$ & $\begin{array}{l}\text { Avg. S/N } \\
\text { Primary }\end{array}$ & $\begin{array}{l}\text { Avg. S/N } \\
\text { Secondary }\end{array}$ \\
\hline \multirow[t]{4}{*}{ 2MASS J07464256+2000321AB } & 2006 Dec 16 & HIP 41798 & 1200 & 4 & 52 & 44 \\
\hline & 2007 Dec 4 & HIP 41798 & 1200 & 6 & 72 & 59 \\
\hline & 2008 Dec 19 & HIP 41798 & 1200 & 6 & 66 & 56 \\
\hline & 2009 Dec 9 & HIP 41798 & 1200 & 2 & 83 & 70 \\
\hline \multirow[t]{2}{*}{ 2MASS J14263161+1557012AB } & 2007 Jun 8 & HIP 73087 & 1200 & 4 & 44 & 33 \\
\hline & 2009 Jun 12 & HIP 73087 & 1200 & 4 & 41 & 29 \\
\hline \multirow[t]{3}{*}{ 2MASS J17501291+4424043AB } & 2008 May 31 & HIP 87045 & 1200 & 4 & 48 & 36 \\
\hline & 2009 Jun 12 & HIP 87045 & 1200 & 6 & 41 & 31 \\
\hline & 2010 Jun 7 & HIP 87045 & 1200 & 4 & 36 & 26 \\
\hline \multirow[t]{3}{*}{ 2MASS J18470342+5522433AB } & 2007 Jun 8 & HIP 93713 & 1200 & 4 & 69 & 60 \\
\hline & 2008 Jun 1 & HIP 93713 & 1200 & 5 & 69 & 60 \\
\hline & 2009 Jun 13 & HIP 93713 & 1200 & 3 & 39 & 36 \\
\hline \multirow[t]{4}{*}{ 2MASS J21402931+1625183AB } & 2007 Jun 9 & HIP 108060 & 1200 & 4 & 43 & 28 \\
\hline & 2008 May 31 & HIP 108060 & 1800 & 3 & 58 & 40 \\
\hline & 2009 Jun 13 & HIP 108060 & 1800 & 2 & 38 & 26 \\
\hline & 2009 Dec 10 & HIP 116611 & 1800 & 3 & 56 & 45 \\
\hline \multirow[t]{4}{*}{ 2MASS J22062280-2047058AB } & 2007 Jun 9 & HIP 116750 & 1200 & 3 & 47 & 39 \\
\hline & 2008 Jun 1 & HIP 109689 & 1200 & 4 & 54 & 48 \\
\hline & 2009 Jun 12 & HIP 109689 & 1200 & 4 & 47 & 44 \\
\hline & 2010 Jun 7 & HIP 109689 & 1200 & 4 & 29 & 29 \\
\hline \multirow[t]{3}{*}{ GJ 569Bab } & 2007 Jun 9 & HIP 73087 & 900 & 2 & 89 & 82 \\
\hline & 2009 Jun 13 & HIP 73087 & 900 & 4 & 86 & 67 \\
\hline & 2010 Jun 6 & HIP 73087 & 900 & 4 & 114 & 97 \\
\hline \multirow[t]{3}{*}{ HD 130948BC } & 2007 Jun 9 & HIP 73087 & 1200 & 4 & 43 & 37 \\
\hline & 2010 Jun 7 & HIP 73087 & 1800 & 4 & 59 & 52 \\
\hline & 2011 Jun 18 & HIP 73087 & 1800 & 4 & 52 & 48 \\
\hline \multirow[t]{4}{*}{ LHS 2397aAB } & 2007 Dec 4 & HIP 58188 & 1800 & 2 & 68 & 27 \\
\hline & 2009 Jun 12 & HIP 61318 & 1800 & 2 & 103 & 33 \\
\hline & 2009 Dec 9 & HIP 58188 & 1800 & 3 & 105 & 29 \\
\hline & 2010 Jun 7 & HIP 61318 & 1800 & 2 & 77 & 31 \\
\hline \multirow[t]{5}{*}{ LP 349-25AB } & 2006 Dec 16 & HIP 5132 & 600 & 4 & 58 & 45 \\
\hline & 2007 Dec 4 & HIP 5132 & 900 & 1 & 63 & 58 \\
\hline & 2008 Dec 19 & HIP 5132 & 1200 & 4 & 105 & 84 \\
\hline & 2009 Jun 12 & HIP 5132 & 1200 & 4 & 114 & 98 \\
\hline & 2009 Dec 9 & HIP 5132 & 1200 & 4 & 121 & 107 \\
\hline \multirow[t]{2}{*}{ LP $415-20 \mathrm{AB}$} & 2008 Dec 19 & HIP 24555 & 1200 & 4 & 42 & 32 \\
\hline & $2009 \operatorname{Dec} 9$ & HIP 22845 & 1800 & 2 & 62 & 49 \\
\hline
\end{tabular}

Note. ${ }^{a}$ All data taken before 2009 December represent the same NIRSPAO-LGS data set presented in Konopacky et al. (2010).

measured for each source at each epoch, along with the weighted average of all epochs, is given in Table 3 (all sources were observed at least two times).

In order to confirm that our method returns the correct $v \sin i$ values, we obtained NIRSPAO observations of two previously measured M type stars. These objects, GL 1245A (M5.5V) and G188-38 (M4V), were targeted by several studies in the optical. Mohanty \& Basri (2003) measured projected rotational velocities of $22.5 \pm 3.7 \mathrm{~km} \mathrm{~s}^{-1}$ for GL $1245 \mathrm{~A}$ and $29.4 \pm$ $6.2 \mathrm{~km} \mathrm{~s}^{-1}$ for G188-38. Other measurements for G188-38 include $36.5 \pm 0.3 \mathrm{~km} \mathrm{~s}^{-1}$ by Donati et al. (2006) and $29.4 \pm$ $1.4 \mathrm{~km} \mathrm{~s}^{-1}$ by Delfosse et al. (1998). We performed an identical analysis to that of our brown dwarf sample on these two mid-M stars, only using a higher temperature template that is more appropriate for these objects. We derived $v$ sin $i$ 's of $19 \pm$ $3 \mathrm{~km} \mathrm{~s}^{-1}$ for GL 1245A and $34 \pm 3 \mathrm{~km} \mathrm{~s}^{-1}$ for G188-38, consistent with all the values from the literature.

As an additional test, we can use "slow rotators" in our sample as templates, artificially spinning them up to estimate the $v \sin i$ of other objects in our sample. Although this method suffers from template mismatch that is remedied by the use of theoretical atmospheres, it offers a further confirmation of our technique. We perform this analysis on one epoch of data for each binary, taken either in 2007 or 2008 . We use the source with the lowest measured $v \sin i$, 2MASS $1847+55 \mathrm{~A}$, as our template for all other objects. This template, corrected for telluric absorption using our observed A0V standards, was "spun up" to produce an artificial grid of spectra with $v \sin i$ between 5 and $100 \mathrm{~km} \mathrm{~s}^{-1}$. The grid was then cross-correlated with each object's spectrum, also corrected for telluric absorption, and we determined the value of $v \sin i$ that provided the maximum correlation. In all cases, the best value for $v \sin i$ found with this technique is within the uncertainties of the values given in Table 3.

Using these two independent checks, we are confident that our methodology is sound and that we are incorporating the necessary uncertainties via our usage of multiple temperature and $\log (g)$ templates. We do caution, however, that objects with 


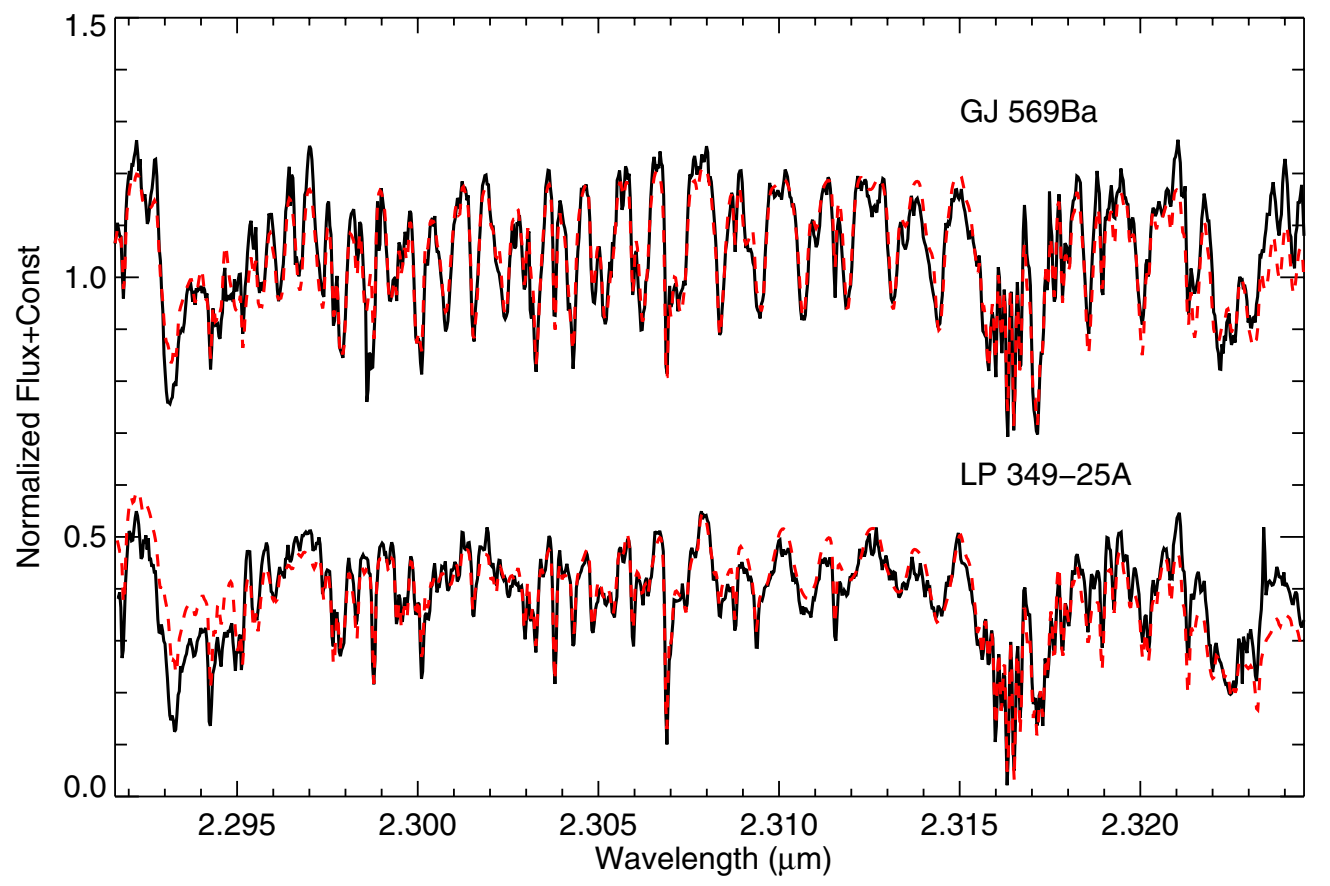

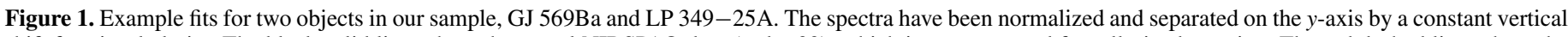

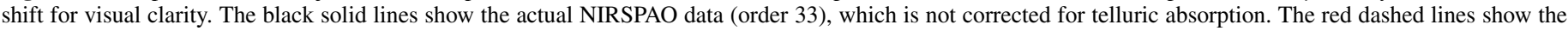

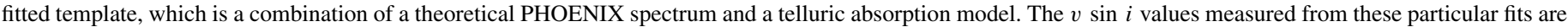
$18 \mathrm{~km} \mathrm{~s}^{-1}$ for GJ 569Ba and $56 \mathrm{~km} \mathrm{~s}^{-1}$ for LP 349-25A. For additional examples of fits for all sources in our sample, see Figure 4 of Konopacky et al. (2010).

Table 3

$v \sin i$ Measurements $\left(\mathrm{km} \mathrm{s}^{-1}\right)$

\begin{tabular}{|c|c|c|c|c|c|c|c|c|c|c|c|c|}
\hline Target & $\begin{array}{l}\text { Sp. } \\
\text { Type }\end{array}$ & $\begin{array}{l}\text { Adopted } \\
T_{\text {eff }}(\mathrm{K})^{\mathrm{a}}\end{array}$ & $\begin{array}{c}2006 \\
\text { December }\end{array}$ & $\begin{array}{l}2007 \\
\text { June }\end{array}$ & $\begin{array}{c}2007 \\
\text { December }\end{array}$ & $\begin{array}{c}2008 \\
\text { May/June }\end{array}$ & $\begin{array}{c}2008 \\
\text { December }\end{array}$ & $\begin{array}{l}2009 \\
\text { June }\end{array}$ & $\begin{array}{c}2009 \\
\text { December }\end{array}$ & $\begin{array}{l}2010 \\
\text { June }\end{array}$ & $\begin{array}{l}2011 \\
\text { June }\end{array}$ & $\begin{array}{l}\text { Weighted } \\
\text { Average }\end{array}$ \\
\hline 2MASS $0746+20 \mathrm{~A}$ & L0 & 2205 & $19 \pm 5$ & $\ldots$ & $18 \pm 5$ & $\ldots$ & $18 \pm 5$ & $\ldots$ & $20 \pm 3$ & $\ldots$ & $\ldots$ & $19 \pm 2$ \\
\hline 2MASS $0746+20 B$ & L1.5 & 2060 & $33 \pm 6$ & $\cdots$ & $32 \pm 6$ & $\cdots$ & $32 \pm 6$ & $\cdots$ & $34 \pm 6$ & $\cdots$ & $\cdots$ & $33 \pm 3$ \\
\hline 2MASS 1426+16A & M8.5 & 2400 & $\cdots$ & $6 \pm 4$ & $\cdots$ & $6 \pm 5$ & $\cdots$ & $7 \pm 4$ & $\cdots$ & $\cdots$ & $\cdots$ & $6 \pm 3$ \\
\hline 2MASS $1426+16 B$ & L1 & 2240 & $\cdots$ & $8 \pm 7$ & $\cdots$ & $12 \pm 4$ & $\cdots$ & $11 \pm 4$ & $\cdots$ & $\cdots$ & $\cdots$ & $11 \pm 3$ \\
\hline 2MASS $1750+44 \mathrm{~A}$ & M7.5 & 2200 & $\cdots$ & $\cdots$ & $\cdots$ & $9 \pm 3$ & $\cdots$ & $10 \pm 4$ & $\cdots$ & $8 \pm 4$ & $\cdots$ & $9 \pm 2$ \\
\hline 2MASS $1750+44 B$ & L0 & 2020 & $\cdots$ & $\cdots$ & $\cdots$ & $10 \pm 4$ & $\cdots$ & $10 \pm 5$ & $\cdots$ & $12 \pm 4$ & $\cdots$ & $11 \pm 3$ \\
\hline 2MASS $1847+55 \mathrm{~A}$ & M7 & 2400 & $\cdots$ & $3 \pm 5$ & $\cdots$ & $4 \pm 4$ & $\cdots$ & $9 \pm 3$ & $\cdots$ & $8 \pm 4$ & $\cdots$ & $7 \pm 2$ \\
\hline 2MASS $1847+55 B$ & M7.5 & 2100 & $\cdots$ & $5 \pm 4$ & $\cdots$ & $5 \pm 5$ & $\cdots$ & $9 \pm 4$ & $\cdots$ & $9 \pm 4$ & $\cdots$ & $7 \pm 2$ \\
\hline 2MASS $2140+16 A$ & M8.5 & 2300 & $\cdots$ & $13 \pm 5$ & $\cdots$ & $12 \pm 4$ & $\cdots$ & $11 \pm 3$ & $16 \pm 4$ & $\cdots$ & $\cdots$ & $13 \pm 2$ \\
\hline 2MASS $2140+16 B$ & L2 & 2075 & $\cdots$ & $42 \pm 7$ & $\cdots$ & $37 \pm 6$ & $\cdots$ & $34 \pm 6$ & $38 \pm 7$ & $\cdots$ & $\cdots$ & $37 \pm 3$ \\
\hline 2MASS 2206-20A & M8 & 2350 & $\cdots$ & $20 \pm 4$ & $\cdots$ & $18 \pm 3$ & $\cdots$ & $19 \pm 4$ & $\cdots$ & $16 \pm 6$ & $\cdots$ & $19 \pm 2$ \\
\hline 2MASS 2206-20B & M8 & 2250 & $\cdots$ & $22 \pm 4$ & $\cdots$ & $20 \pm 4$ & $\cdots$ & $21 \pm 4$ & $\cdots$ & $18 \pm 6$ & $\cdots$ & $21 \pm 2$ \\
\hline GJ 569Ba & M8.5 & 2000 & $\cdots$ & $19 \pm 3$ & $\cdots$ & $\cdots$ & $\cdots$ & $18 \pm 3$ & $\cdots$ & $19 \pm 3$ & $\cdots$ & $19 \pm 2$ \\
\hline GJ 569Bb & M9 & 2000 & $\cdots$ & $5 \pm 5$ & $\cdots$ & $\cdots$ & $\cdots$ & $6 \pm 5$ & $\cdots$ & $7 \pm 4$ & $\cdots$ & $6 \pm 3$ \\
\hline HD 130948B & L4 & 1840 & $\cdots$ & $63 \pm 8$ & $\cdots$ & $\cdots$ & $\cdots$ & $\cdots$ & $\cdots$ & $61 \pm 7$ & $62 \pm 6$ & $62 \pm 4$ \\
\hline HD $130948 C$ & L4 & 1790 & $\cdots$ & $86 \pm 8$ & $\cdots$ & $\cdots$ & $\cdots$ & $\cdots$ & $\cdots$ & $87 \pm 11$ & $84 \pm 12$ & $86 \pm 6$ \\
\hline LHS 2397aA & M8 & 2180 & $\cdots$ & $\cdots$ & $14 \pm 3$ & $14 \pm 3$ & $15 \pm 3$ & $15 \pm 3$ & $15 \pm 3$ & $15 \pm 3$ & $\cdots$ & $15 \pm 1$ \\
\hline LHS 2397aB & L7.5 & 1350 & $\cdots$ & $\cdots$ & $10 \pm 6$ & $10 \pm 6$ & $14 \pm 6$ & $10 \pm 8$ & $10 \pm 6$ & $11 \pm 8$ & $\cdots$ & $11 \pm 3$ \\
\hline LP $349-25 A$ & M8 & 2200 & $56 \pm 5$ & $\cdots$ & $50 \pm 11$ & $\cdots$ & $59 \pm 7$ & $55 \pm 4$ & $54 \pm 4$ & $\cdots$ & $\cdots$ & $55 \pm 2$ \\
\hline LP $349-25 B$ & M9 & 2050 & $87 \pm 6$ & $\cdots$ & $79 \pm 11$ & $\cdots$ & $76 \pm 10$ & $81 \pm 4$ & $85 \pm 5$ & $\cdots$ & $\cdots$ & $83 \pm 3$ \\
\hline LP $415-20 A$ & M7 & 2300 & $\cdots$ & $\cdots$ & $\cdots$ & $\cdots$ & $40 \pm 6$ & $\cdots$ & $41 \pm 7$ & $\cdots$ & $\cdots$ & $40 \pm 5$ \\
\hline LP $415-20 B$ & M9.5 & 2000 & $\cdots$ & $\cdots$ & $\cdots$ & $\cdots$ & $36 \pm 4$ & $\cdots$ & $40 \pm 7$ & $\cdots$ & $\cdots$ & $37 \pm 4$ \\
\hline
\end{tabular}

Note. ${ }^{\text {a }}$ From Konopacky et al. (2010). To be conservative, our analysis assumed a $\pm 300 \mathrm{~K}$ temperature uncertainty for all objects.

particularly high values of $v \sin i$ ( $>40 \mathrm{~km} \mathrm{~s}^{-1}$; four objects in our sample), though undoubtedly rapid rotators, might be subject to additional systematic uncertainties not fully accounted for in our analysis due to greater sensitivity to properties associated with the instrument and technique, and this uncertainty may not be captured in the averaged values in Table 3.

We also estimate the lowest measurable value of $v \sin i$ in our spectra. To do this, we took our PHOENIX templates and broadened them first to the correct instrumental profile and then to different values of $v \sin i$. We also injected random Gaussian noise such that the templates would have $\mathrm{S} / \mathrm{N} \sim 55$, which is the average effective $\mathrm{S} / \mathrm{N}$ per pixel for our data. We fit these spectra using the method described above. We find that the limiting value for which we could accurately measure $v \sin i$ is $3 \mathrm{~km} \mathrm{~s}^{-1}$. Note that we are able to measure $v \sin i$ below the intrinsic resolution of our NIRSPAO data due to our 


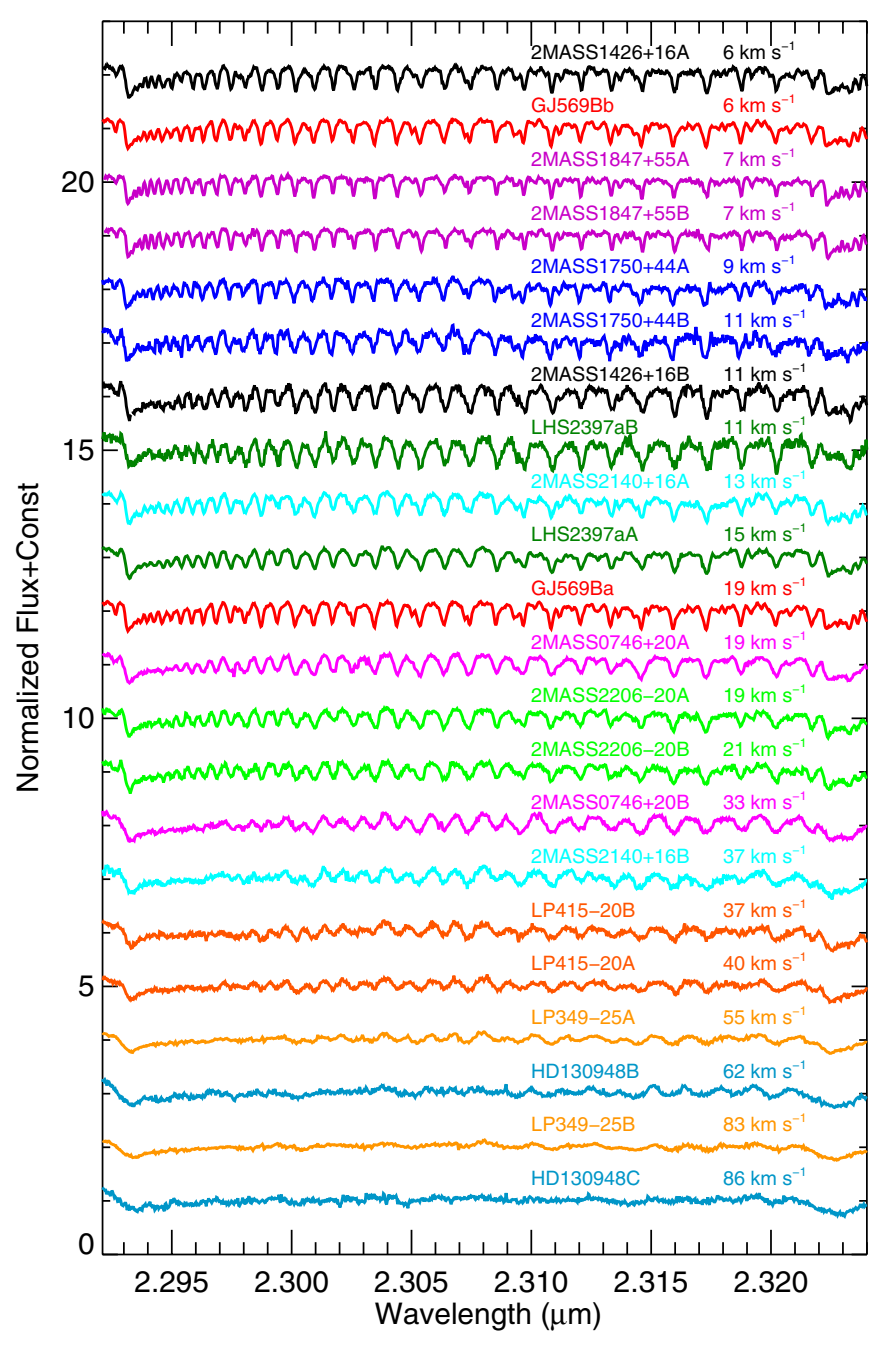

Figure 2. Single epoch examples of spectra for all objects in our sample (NIRSPAO order 33). All spectra have been normalized to the continuum and shifted to the same radial velocity. The spectra have been separated on the $y$-axis by a constant vertical shift, such that the absolute value of the flux is arbitrary. Sources are arranged in order of increasing rotational velocity and color-coded such that components in the same system have matching colors. Increasing rotational velocity dramatically impacts the morphology of the $\mathrm{CO}$ band head, which falls near the end of the $K$ band at $\sim 2.3 \mu \mathrm{m}$.

accurate measurement of the instrumental profile and theoretical templates that closely match our actual spectra.

Figure 2 shows example spectra for all sources in our sample. These spectra, which we have corrected for telluric absorption for plotting purposes, are arranged in order of increasing $v \sin i$, demonstrating the effect of rotational velocity on $\mathrm{CO}$ band head morphology.

\section{DISCUSSION}

This study represents the first measurement of component rotational velocities for a large sample of VLM binaries. The values presented in Table 3 show that $\sim 80 \%$ of our sample are rapid rotators $\left(v \sin i \gtrsim 10 \mathrm{~km} \mathrm{~s}^{-1}\right)$, and two sources, LP 349-25B and HD130948C, are among the fastest rotating VLM objects ever observed. In this section, we discuss the implications of these measurements.

\subsection{Comparison to $v \sin i$ Measurements in the Literature}

In Figure 3, we plot our measured $v$ sin $i$ 's as a function of spectral type. We also include $v \sin i$ measurements from the literature, derived from seeing-limited observations (Mohanty \& Basri 2003; Zapatero Osorio et al. 2006; Reiners \& Basri 2008, 2010; Blake et al. 2010) with comparable sample, spectral resolution, and spectral type coverage to our observations. Our measurements are consistent with previous observations, which also find that VLM objects tend to be rapid rotators. In addition, our results are consistent with the trend of increasing $v \sin i$ with later spectral type. In a number of cases, the values we measure are higher than those objects presented in the literature of a given spectral type. This is likely attributable to the mixed ages probed in this study. Reiners \& Basri (2008) show that rotational velocity is a function of the age of the system, correlated with a spin-down timescale that is driven by magnetic braking. In our sample, for instance, we find that the $v \sin i$ measurements for HD 130948BC are higher than all previous measurements for mid-L dwarfs, and it has been proposed that this system is younger than the majority of the field population ( 400-800 Myr; Dupuy et al. 2009b; Mullan $\&$ MacDonald 2010). Indeed, the extremely rapid rotation of HD 130948BC may imply that the younger age of the system preferred by Mullan \& MacDonald (2010) is more plausible. Our other extremely rapidly rotating system, LP349-25AB, has also been proposed to be quite young ( $\sim 140$ Myr; Dupuy et al. 2010). On the whole, however, the measurements in our sample are fully consistent with the bulk population of objects previously observed.

We also note that with these very rapid rotation rates, both LP 349-25B and HD 130948C are rotating at $\sim 30 \%$ of their break-up speed. These large rotational velocities should also cause a high degree of rotational flattening. Using Equation (6) of Barnes \& Fortney (2003), we derive that the ratio of the polar radii to the equatorial radii should be $\sim 0.87$. Given this level of oblateness, these objects might be expected to exhibit measurable linear polarization (Sengupta \& Marley 2010). In addition, the cooler temperatures at the equator due to gravity darkening may affect the spectral type measurements, as demonstrated by surface imaging of rapidly rotating intermediate-mass stars (Monnier et al. 2007). The rapid rotation also leads to a higher level of uncertainty in our radial velocity measurements for these extremely rapidly rotating sources. As is apparent in Figure 2, the majority of the $\mathrm{CO}$ band head features are basically smoothed by the rapid rotation, making anchoring these objects precisely in wavelength space quite challenging and giving rise to the relatively high radial velocity uncertainties given in Konopacky et al. (2010).

Several sources in our sample were previously targeted in studies that did not resolve the components but did measure $v$ sin $i$. LP $349-25 \mathrm{AB}$, LHS 2397aAB, and 2MASS 2206-20AB were observed by Reiners \& Basri (2010). They obtained values consistent with ours for 2MASS 2206-20AB and the primary component of LP 349-25AB, but a very different value for LHS 2397aAB. We are not certain why our $v \sin i$ measurement of LHS 2397aAB is different from these authors, but speculate that perhaps it can be attributed to the broadening of the lines due to the binary orbit in the unresolved spectra. Jones et al. (1996) measured the unresolved $v \sin i$ for LP 415-20AB, also finding a result consistent with our measurements (for both components). Blake et al. (2010) measured an unresolved $v \sin i$ for 2MASS $0746+20 \mathrm{AB}$. They obtain a nearly identical value to what we measure for $2 \mathrm{MASS}$ $0746+20 \mathrm{~B}$, although the combined light of the system should be dominated by the primary. However, the flux ratio of $\sim 1.4$ at the $K$ band means the dominance is not extreme. 


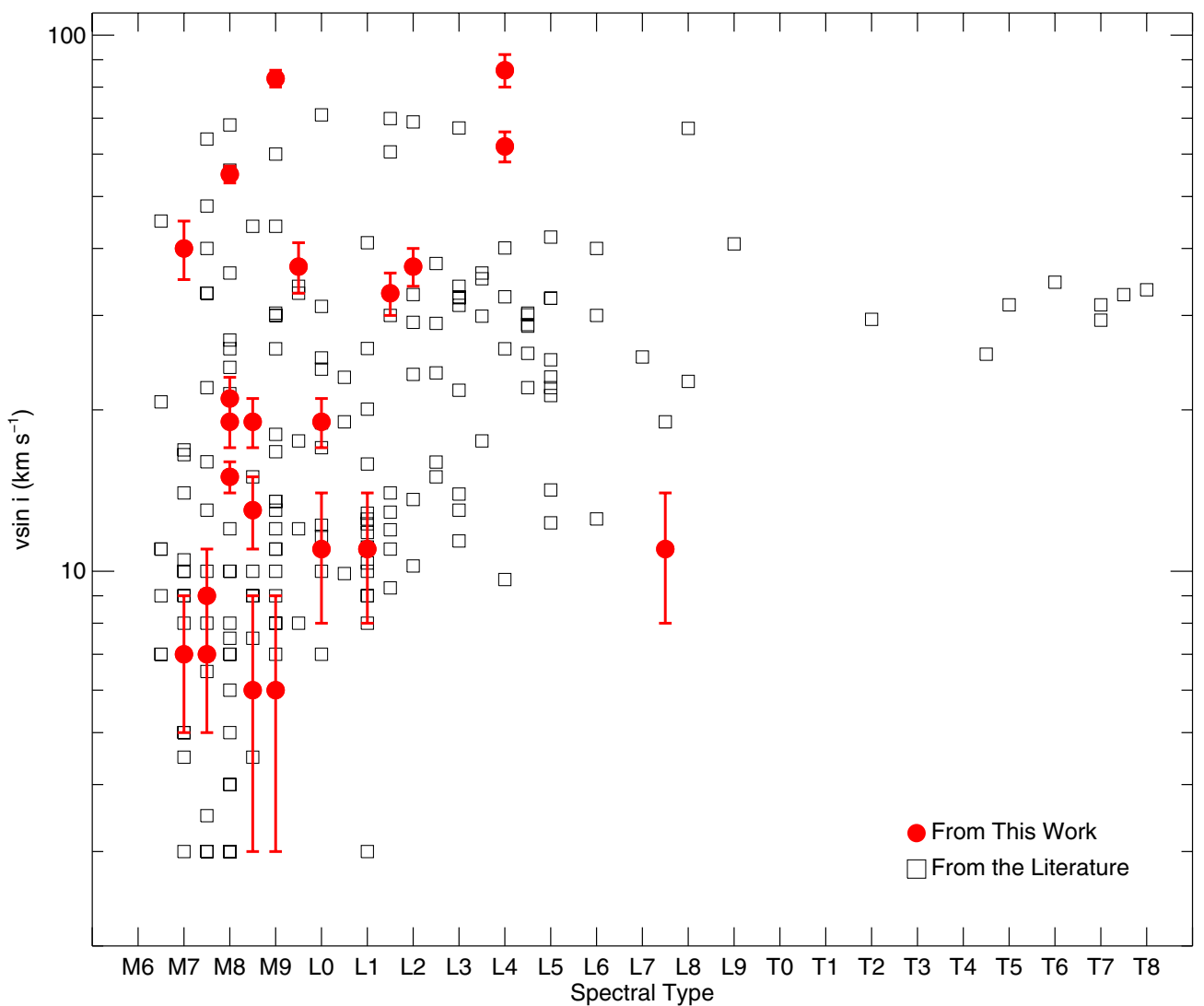

Figure 3. Projected rotational velocity $(v \sin i)$ vs. spectral type for each of the binary components in our sample (red circles). Also plotted (open squares; uncertainties omitted for clarity) are previously measured values (seeing-limited observations, so binaries are not resolved) of $v$ sin $i$ for field VLM objects in the literature (Mohanty \& Basri 2003; Zapatero Osorio et al. 2006; Reiners \& Basri 2008, 2010; Blake et al. 2010). The values we measure for our sources are consistent with other VLM objects, with our sources tending toward rapid rotation $\left(v \sin i>10 \mathrm{~km} \mathrm{~s}^{-1}\right)$.

(A color version of this figure is available in the online journal.)

\subsection{Component $v$ sin $i$ Comparison}

We can also compare the rotational velocities of the components in each system to each other. The results of this comparison are shown in Figure 4. It is immediately apparent that a number of components in the same system have vastly different $v \sin i$. There are five binaries in our sample that exhibit statistically significantly $(>3 \sigma)$ differing $v$ sin $i$. These five systems, 2MASS 0746+20AB, 2MASS 2104+16AB, GJ 569Bab, HD 130948BC, and LP 349-25AB, have differences $>10 \mathrm{~km} \mathrm{~s}^{-1}$, or $>30 \%$. For all but GJ 569Bab, the secondary appears to be rotating more rapidly than the primary. For the other six systems in our sample, the velocities are consistent to within $2 \sigma$. We also note that the consistency of these six systems, plus the generally correlated rapid or slow rotation of the five systems with different velocities, implies that the components of VLM binaries are not randomly paired in $v \sin i$.

Simon et al. (2006) noted that the lines of their spatially resolved spectra were broader for GJ 569Ba than for GJ 569Bb. They measured $v$ sin $i$ 's of $25 \mathrm{~km} \mathrm{~s}^{-1}$ and $10 \mathrm{~km} \mathrm{~s}^{-1}$ for Ba and $\mathrm{Bb}$, respectively, close to the values we derive here. The broadening of GJ 569Ba was postulated to perhaps be due to an unresolved third component rather than an intrinsic difference from GJ $569 \mathrm{Bb}$. Given that we see four other systems with differing component $v \sin i$, it is even more plausible that this system does not have an unresolved third component. In addition, Zapatero Osorio et al. (2004) measure $v \sin i$ 's of $37 \mathrm{~km} \mathrm{~s}^{-1}$ and $30 \mathrm{~km} \mathrm{~s}^{-1}$ for $\mathrm{Ba}$ and $\mathrm{Bb}$, thus also noticing a difference in the component values. The higher $v \sin i$ values

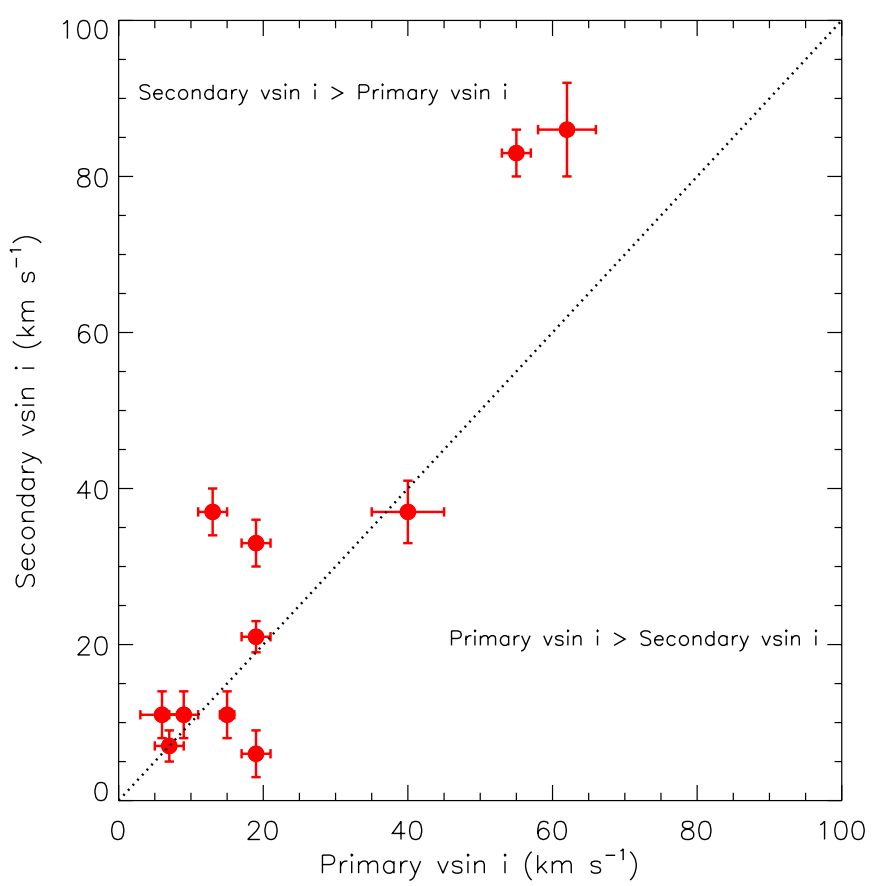

Figure 4. $v$ sin $i$ of each secondary component plotted against its primary. Sources with consistent velocities should fall on the dotted line. Five of our eleven systems show components with $v \sin i$ that differ by $>3 \sigma$. Of those five systems, four have secondary components with higher velocities than their primaries.

(A color version of this figure is available in the online journal.) 

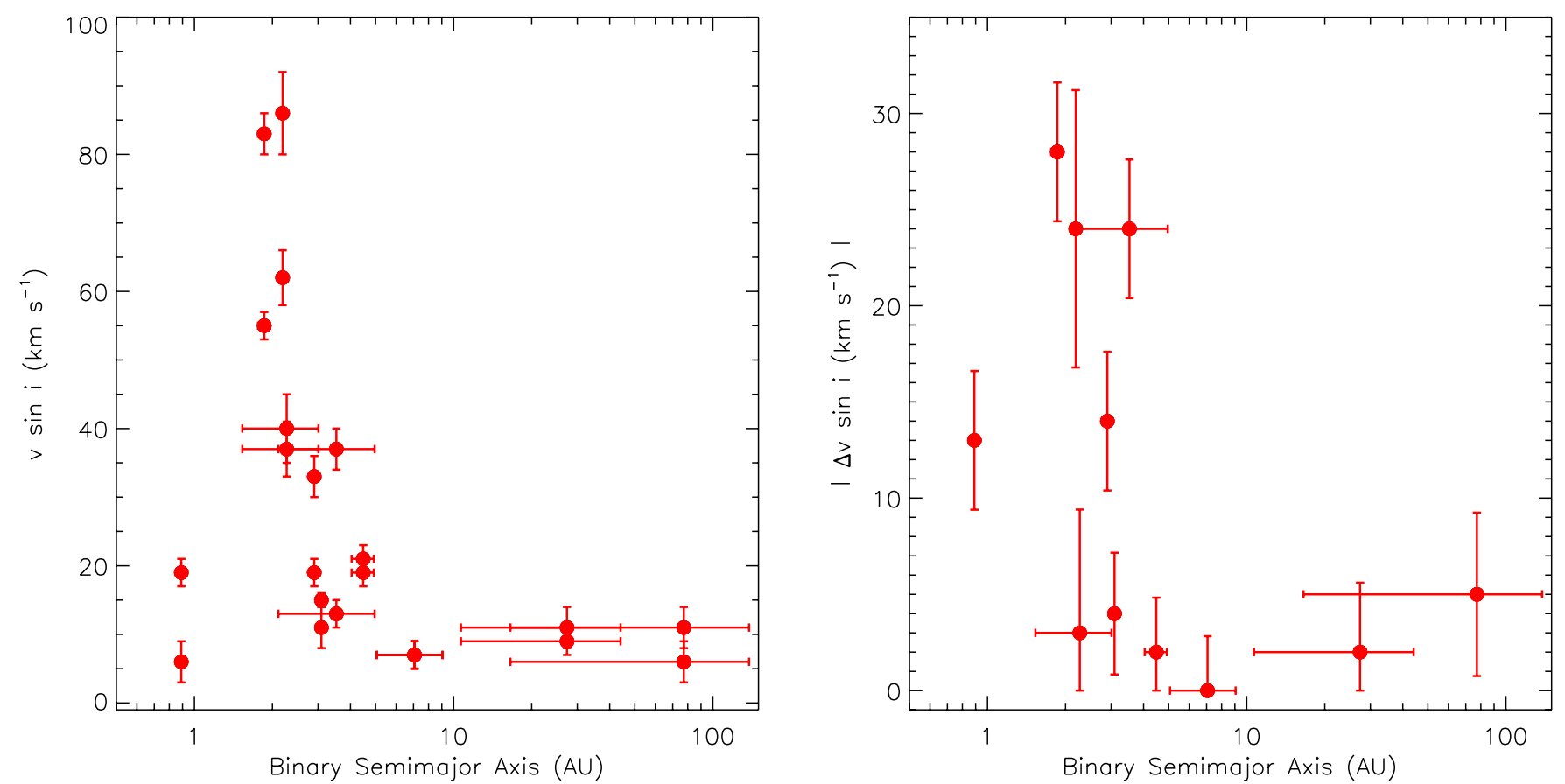

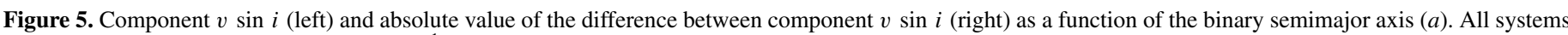

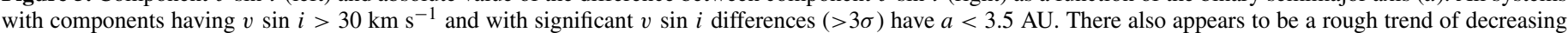

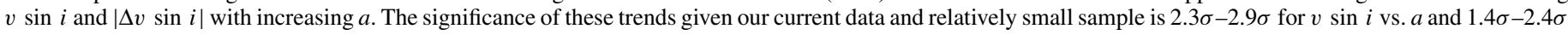
for $|\Delta v \sin i|$ vs. $a$.

(A color version of this figure is available in the online journal.)

potentially stems from their use of KI features in the $J$ band that are known to be gravity sensitive. Although in many cases the radial velocity uncertainties for these sources are quite high (Konopacky et al. 2010), we do not see strong evidence for additional radial velocity variability in any of these discrepant systems that would point obviously to an additional unresolved component.

We note that in the case of HD 130948BC, Mullan \& MacDonald (2010) postulated that the components may not be rotating at the same rate, which we have now shown may be the case, although in contrast to their predictions, the secondary is likely the more rapid rotator and hence potentially the more magnetically active component. However, as we do not in fact know the true equatorial velocity $\left(v_{\text {eq }}\right)$ of either component, we cannot make any definitive statements about their models, although the rapid rotation of both components suggests that magnetic activity could be significant for both. See Section 3.4 for additional discussion of this system.

The targets in this sample all have orbital parameter estimates from previous works (Konopacky et al. 2010; Zapatero Osorio et al. 2004; Simon et al. 2006; Dupuy et al. 2009a, 2009b, 2009c, 2010). We therefore explore the potential impact of the binary orbital properties on the consistency of $v \sin i$. For the purposes of this paper, we use orbital elements derived in Konopacky et al. (2010). We looked for trends in both $v \sin i$ and $\Delta v \sin i$ as a function of all orbital parameters, and the only variable that produces a noticeable trend is semimajor axis (a). All components with $v \sin i>30 \mathrm{~km} \mathrm{~s}^{-1}$ and all five systems with significantly different $v \sin i$ have $a \lesssim 3.5$ AU. In Figure 5, we plot both the component $v \sin i$ and $|\Delta v \sin i|$ versus $a$. To assess the significance of the apparent trend of decreasing velocity and velocity difference with increasing $a$, we use the Spearman's rank correlation coefficient. After accounting for the uncertainties in $v \sin i$ and $a$ via Monte Carlo simulation, we determine using this metric that the significance of the trend in $v \sin i$ is between $2.3 \sigma$ and $2.9 \sigma$ and the significance in $|\Delta v \sin i|$ is between $1.4 \sigma$ and $2.4 \sigma$. Because of the relatively small size of our sample, we are unable to explore the significance of this trend in greater detail. However, we note it here as a possible relationship of interest in the context of the discussion below. In addition, this trend is similar to what was seen in Patience et al. (2002), who observed that tighter binaries in $\alpha$ Per were rotating more rapidly than wider binaries. However, such a trend was not observed by Bouvier et al. (1997) in the Pleiades.

Given these result for the five "discrepant" systems, we are confronted with two possible scenarios. Either the binary components in some systems truly rotating at significantly different rates, or their rotation axes are inclined with respect to each other and possibly their orbital plane (or some combination of these two). We explore these possibilities below in Sections 3.3 and 3.4 .

\subsection{Intrinsic Rotational Velocity Differences?}

It is possible that the binaries in our sample with "discrepant" $v \sin i$ have parallel rotation axes but differing rotational velocities due to intrinsic processes at work during either their formation or early evolution. Binary systems are generally thought to form via fragmentation of a molecular cloud core or large circumstellar disks wherein small seeds are formed that eventually accrete more material, form a disk, and achieve dynamical stability (e.g., Bonnell et al. 1991; Shu et al. 1990). Although the formation mechanism for VLM objects is still an open question, a fragmentation origin is certainly plausible for these objects. Simulations of core fragmentation originally assumed that the rotation axes of the binary seeds were aligned with the rotation axis of the core (Bate 1997). In these simulations, it was shown 
that the properties of the specific angular momentum of the accreting material onto the protobinary had a substantial impact on the properties of the binary. For a binary to grow to a mass ratio of about 1 , as in the case of most of the objects in our sample, requires a higher specific angular momentum for the accreting material, which in turn leads to a higher accretion rate for the secondary component than the primary (Bate 1997). The fraction of this angular momentum that is converted into orbital angular momentum versus spin angular momentum depends on the size of the accretion radius. It has been shown, however, that in cases where significant spin increase is achieved from accretion, the primary component tends to increase more than the secondary component (Bate 1997; Artymowicz 1983), which is not in agreement with most of our "discrepant" systems. In addition, if these objects formed circumstellar disks after the initial accretion phase, they may be subjected to braking through magnetic coupling with the disks, as discussed below.

As has been demonstrated in other works and discussed earlier, there is a clear evolution of rotational velocity as a function of age for VLM objects, much like what is observed for higher mass stars (Reiners \& Basri 2008). It has been suggested that during the pre-main-sequence phase of evolution, rotation is likely regulated by magnetic coupling to a circumstellar disk (e.g., Edwards et al. 1993; Kundurthy et al. 2006), although this mechanism is still a matter of debate (e.g., Stassun et al. 1999; Nguyen et al. 2009). Once the disk dissipates in $\sim 1-10 \mathrm{Myr}$ (Strom et al. 1993), a star or VLM object will speed up due to its continued contraction. It has been shown that circumstellar disks exist around components of binary stars (e.g., McCabe et al. 2006; Cieza et al. 2009). If component disks dissipate on different timescales, one object will begin to speed up sooner than the other. This could potentially lead to differing rotational velocities in spite of coevality. Observational support for this possibility exists via measurements of disks around $\mathrm{T}$ Tauri binaries. For instance, McCabe et al. (2006) identify six $\mathrm{T}$ Tauri binary systems that have component disks in different phases of evolution, with the secondary tending to have the more evolved disk (albeit for slightly higher mass objects than observed in this work). However, it is unclear if a dissipation timescale difference, which would likely be at most around $10 \mathrm{Myr}$, is alone sufficient to generate a large rotational velocity difference in these systems. Furthermore, the tight physical separations of the binaries in our sample should have impacted the formation and survival of any circumstellar disks, leading to both components having disks that last for at most 1 Myr (e.g., Cieza et al. 2009; Duchêne 2010). The truncated disk survival time in close binaries could be related to the tentative trend we see in $v \sin i$ as a function of $a$ in Figure 5.

After the disk dissipates, VLMs likely follow a slightly modified form of the wind braking law that has been shown to reproduce the rotation versus age correlation seen among higher mass objects. The timescale for the braking of these objects is significantly longer than for high-mass objects. In all cases, the rotational speed seems to be clearly a function of mass, which in a coeval system is correlated with spectral type. It could be postulated, therefore, that the reason we observe some secondaries rotating more rapidly than primaries is due to their lower mass/later spectral type. Indeed, Reiners et al. (2007) identify this as the cause of the $v \sin i$ differences in the components of the triple system LHS 1070. In order to determine whether this is a viable cause of the differences in $v \sin i$ we observe for our binaries, we attempt to roughly assess the expected $v \sin i$ as a function of spectral type for a given age.
We use the relationship given in Reiners \& Basri (2008), which uses the wind braking law to describe the rotational velocity evolution of VLM objects, for an age of 2 Gyr. We choose this age because, although the ages of the sources in our sample are generally unknown, it is roughly the average age of the systems in our sample that are thought to be relatively young $(\lesssim 500 \mathrm{Myr})$ and those that are thought to be the age of the field (possibly as old as $\sim 5 \mathrm{Gyr}$ ). Using this relationship, we calculate the expected $v \sin i$ for each binary component based on its spectral type, and then compute the expected difference between the two. We also assume an intrinsic scatter at a given spectral type to account for some of the spread seen in measurements of rotation rates in clusters with known ages (i.e., Terndrup et al. 2000; Irwin et al. 2009). We assume a conservative intrinsic scatter per object of $20 \%$, which gives an uncertainty of between $\sim 7$ and $13 \mathrm{~km} \mathrm{~s}^{-1}$ in $\Delta v \sin i$. Because of the fairly substantial intrinsic scatter assumed here, our choice of using the $2 \mathrm{Gyr}$ relationship from Reiners \& Basri (2008) has little impact on this comparison because values of $\Delta v \sin i$ from their 2,5 , and $10 \mathrm{Gyr}$ relationships are all consistent given these uncertainties except in the case of LHS 2397aAB. For this system, older ages predict an even greater difference in $\Delta v \sin i$, whereas we observe no statistically significant difference in $v \sin i$ in this system. The only assumption that could potentially yield $\Delta v \sin i$ consistent with our measurement is a very young age, which is most likely not the case for this system (Freed et al. 2003; Dupuy et al. 2009c). We plot the values we derive for expected $\Delta v \sin i$ in Figure 6, along with our actual measurements for each binary. While this effect, given our assumptions, could explain the differences in the component velocities in 2 MASS $0746+20 \mathrm{AB}$ and 2 MASS $2140+16 \mathrm{AB}$ (marginally), it gives results inconsistent with our measurements for GJ 569Bab (1.6 $\sigma$ off), HD 130948BC (1.6 $\sigma$ off), or LP $349-25 \mathrm{AB}(2.9 \sigma$ off). And, as mentioned, it predicts a large velocity difference for the components of LHS $2397 \mathrm{aAB}$, which we do not observe. It is also worth noting that GJ 569Bab, HD 130948BC, and LP 349-25AB have all been postulated to be fairly young ( $\lesssim 700$ Myr; Dupuy et al. 2009b, 2010; Simon et al. 2006; Zapatero Osorio et al. 2006).

\subsection{Mutually Inclined Rotation Axes?}

If we instead assume that the binary components with similar masses must have similar or the same rotational velocities, then their rotation axes must be inclined with respect to one another in systems with differing $v \sin i$. To determine the most likely value of the relative inclination of the spin axes in these five systems, we ignore for a moment the known orbital inclination and perform a Monte Carlo simulation assuming that one component has an inclination sampled from a distribution that is uniform in $\cos (i)$. We select a value of $v \sin i$ for each component from a Gaussian distribution defined by our measurements and uncertainties, and assign the randomly sampled inclination to the faster rotating component to determine the equatorial velocity $\left(v_{\text {eq }}\right)$ for the system. We then calculate the inclination required for the other component to have the same $v_{\text {eq. }}$. We perform this exercise 100,000 times to derive probabilities, which we plot in Figure 7. Note that there are two possible values for the component with "unknown" inclination that will give $v_{\mathrm{eq}}$ due to a $180^{\circ}$ ambiguity (axis pointing up or down from our line of sight). Figure 7 shows joint probability density functions (PDFs) between $v_{\text {eq }}$ and the absolute value of the difference in the inclination of the two 


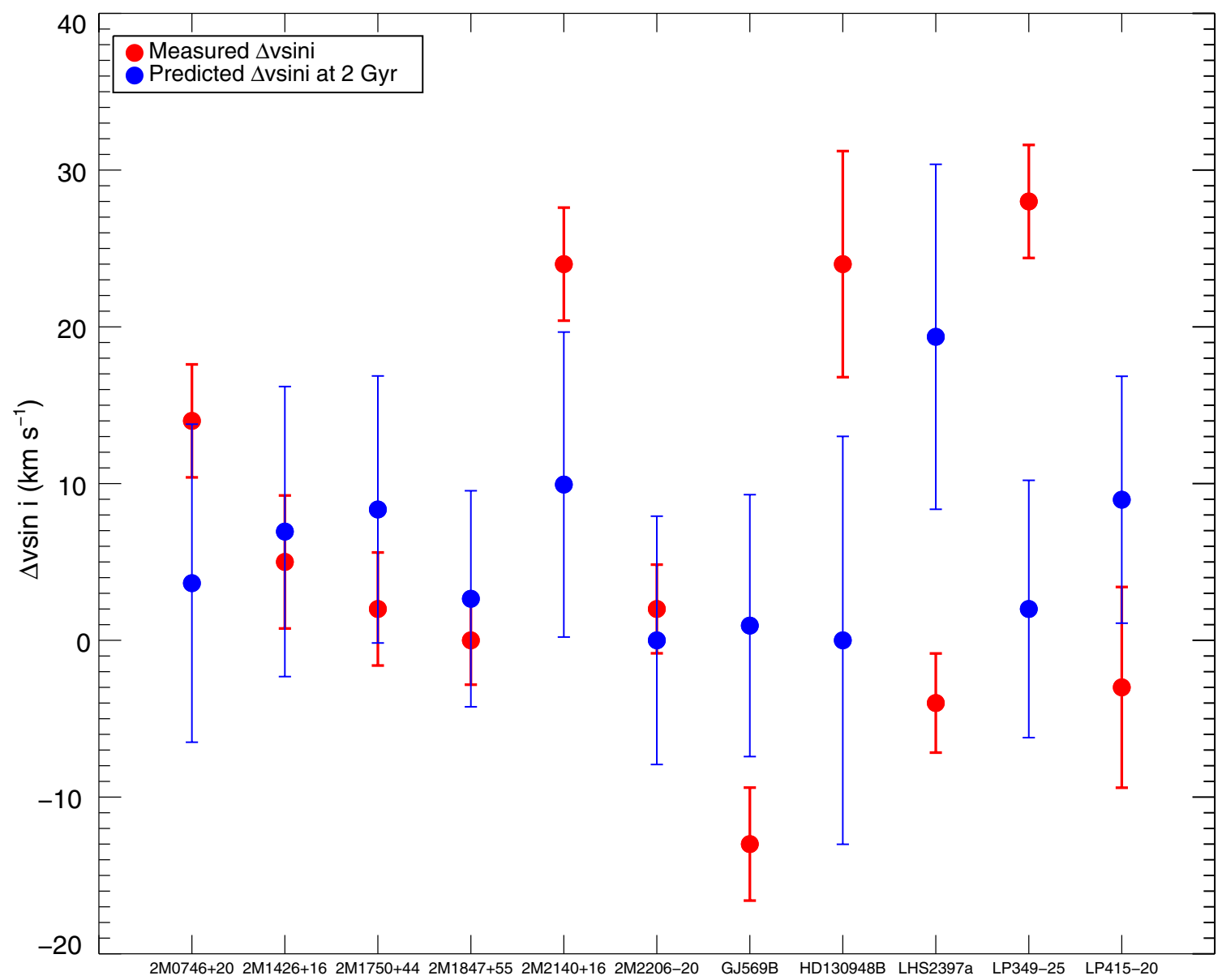

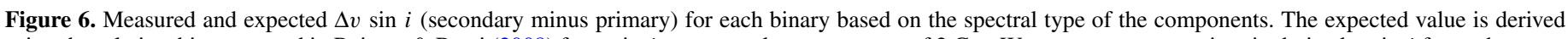

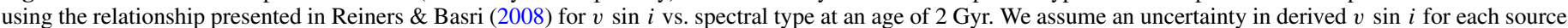

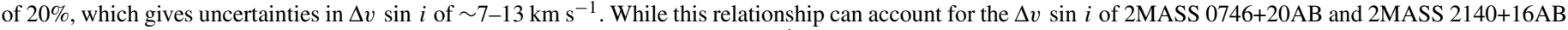
(marginally), the values of GJ 569Bab, HD 130948BC, and LP 349-25AB are all $~ 1.6 \sigma$ discrepant.

components. The white dot overplotted on each distribution denotes the location of peak probability. These peaks are found between $\sim 40$ and $\sim 60 \mathrm{deg}$ in $\Delta i$ and fall close to a $v_{\text {eq }}$ that equals the $v \sin i$ of the faster rotating component since a randomly oriented object is more likely to be observed edge-on than poleon. The figure also demonstrates that the only configuration that can maintain both the same inclination and $v_{\text {eq }}$ is one in which both components rotate close to break-up speed $\left(\sim 300 \mathrm{~km} \mathrm{~s}^{-1}\right)$ and are observed almost pole-on. This is an extremely unlikely configuration and can safely be ruled out as an explanation for all the systems with discrepant $v \sin i$.

It is possible that some level of rotation axis misalignment is natural in these binary systems and could be represented by a Gaussian distribution centered on some "typical" misalignment. We can use our sample to assess this toy model assuming that the components have the same $v_{\text {eq }}$. In order to determine the value for the typical relative inclination and its $1 \sigma$ spread, we performed another Monte Carlo simulation in which we sampled a relative inclination for each system from the distribution allowed by our $v \sin i$ measurements and uncertainties. In this case, however, we assumed that one component was aligned with the orbital plane, sampling from a distribution allowed by the measurements of orbital inclination (Konopacky et al. 2010). These inclination distributions tend to avoid cases with very high $v_{\text {eq }}$ and therefore are more realistic. As before, we assigned the more rapidly rotating component the sampled inclination, and calculated the inclination required for the other component to have the same $v_{\text {eq. }}$. We then generated many $(100,000)$ distributions of relative inclinations for our sample, which we fit with a simple Gaussian model to find the peak and FWHM. We performed the simulation for two cases, one in which we chose the smaller of the two allowed relative inclinations (due to the $180^{\circ}$ ambiguity), and the other choosing at random either the smaller or the larger of the allowed relative inclinations. In the first case (small angles), we find that the preferred distribution has the form $18^{\circ} \pm 26^{\circ}$ and in the second case (any angle) the distribution has the form $25^{\circ} \pm 60^{\circ}$. The implication of this is that if we are truly probing a Gaussian distribution of relative inclinations with the expectation of a few many-sigma outliers, the average inclination and spread must be quite substantial in order to describe for our sample.

The idea of forming objects in which the rotation axis is inclined with respect to the orbital axis has been explored in great detail recently due to the discovery of planets orbiting in a plane that is misaligned with the stellar rotation axis (e.g., Winn et al. 2009, 2010; Triaud et al. 2010). For instance, Bate et al. (2010) investigated the accretion history of stars forming in a turbulent cluster environment. Given variable accretion rates and material being accreted from different directions, it is possible to impact both the rate of rotation and the axis of rotation, leading to rotation axes that are misaligned with circumstellar disks. Alternatively, Lai et al. (2011) have explored how the 

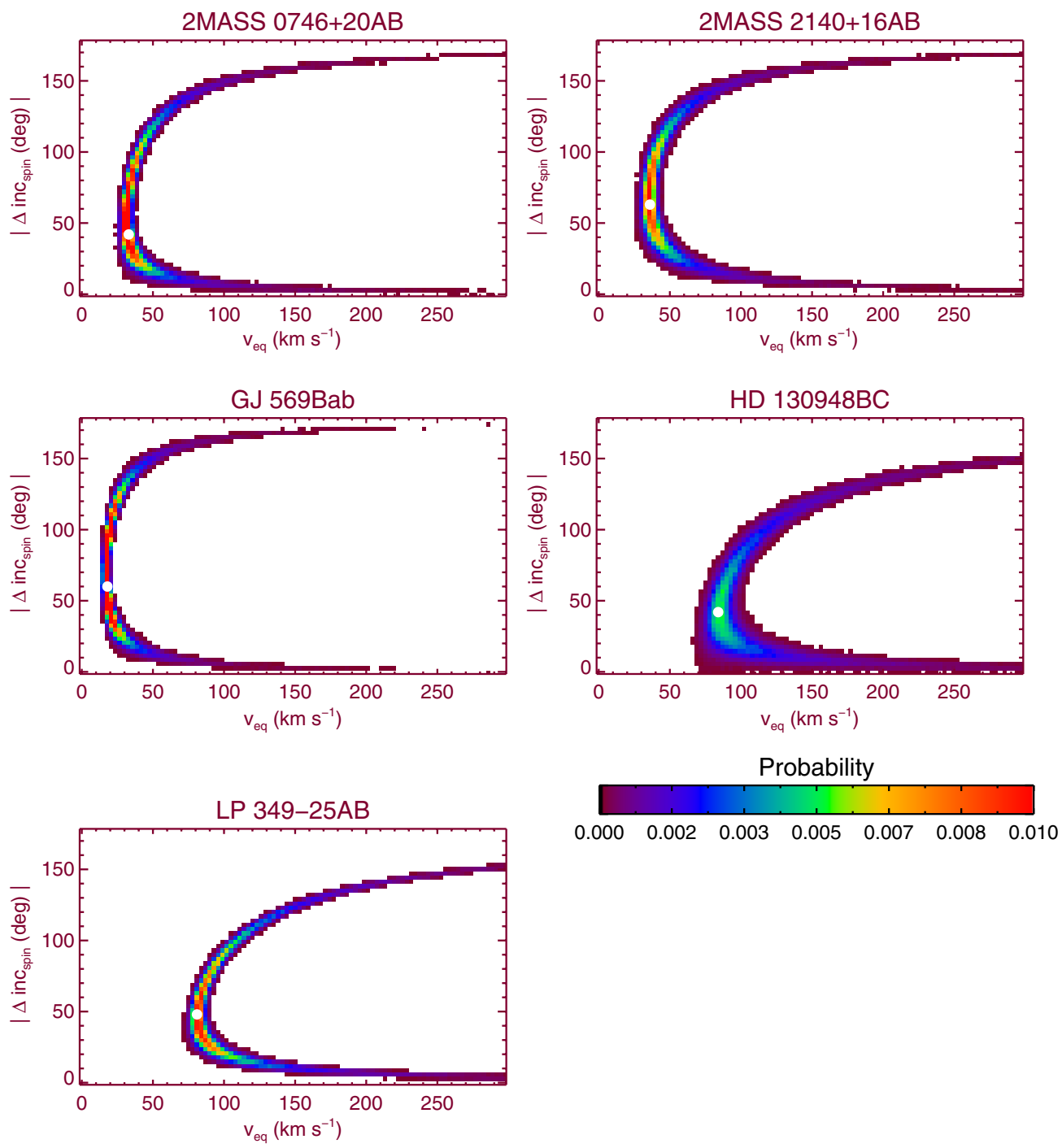

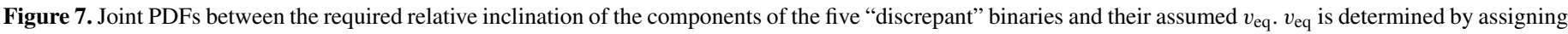

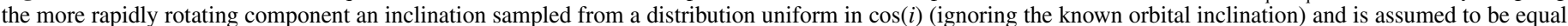

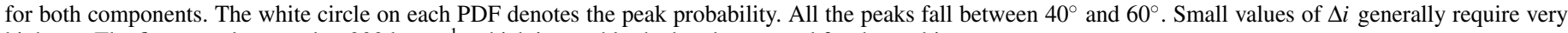
high $v_{\text {eq }}$. The figures only extend to $300 \mathrm{~km} \mathrm{~s}^{-1}$, which is roughly the break-up speed for these objects.

interaction between the magnetic field of a young star and its circumstellar disk can effectively push the stellar rotation axis out of alignment due to a magnetic warping torque. Such scenarios could potentially cause binary stars to have inclined rotation axes.

A third possibility is secular torques due to a third stellar companion. Indeed, Hale (1994) gave a general rule that binary components closer than 40 AU have agreement in spin orientation, but mentioned that rule is broken for systems with a third body. In particular, consider a binary star that is born with each of its component spin vectors aligned with the orbital angular momentum. If the orbital orientation remains fixed throughout the binary's lifetime, there would be no torque to cause spin precession, and the components would remain aligned with the orbit. However, if a companion star $\left(m_{3}\right)$ is introduced on an external, non-coplanar orbit of period $P_{\text {out }}$ and inclination $i$ relative to the binary $\left(m_{1}-m_{2}\right)$ of period $P_{\text {in }}$, it will cause orbital nodal precession in the binary on a secular period

$$
T_{\mathrm{sec}} \approx \frac{4}{3 \cos i} \frac{P_{\mathrm{out}}^{2}}{P_{\mathrm{in}}} \frac{m_{1}+m_{2}+m_{3}}{m_{3}}
$$

(Kiseleva et al. 1998). In response to this misaligned orbit, the spins will precess as well, on a period

$$
T_{\text {precess }, 1}=P_{1} \frac{m_{1}}{m_{2}} \frac{C_{1}}{k_{2,1}}\left(\frac{a}{R_{1}}\right)^{3} \frac{\left(1-e^{2}\right)^{3 / 2}}{\cos \psi}
$$

(Eggleton \& Kiseleva-Eggleton 2001) for star 1, where $k_{2,1}$ is the apsidal motion constant $k_{2}$ for star $1, C_{1}$ is its normalized inertia $\left(=I / M R^{2}\right), P_{1}$ is its spin period, $\psi$ is its spin obliquity, and $a$ and $e$ are the semimajor axis and the eccentricity of the orbit. An analogous period for $m_{2}$ need not be the same if the masses and radii are not quite equal. Thus, we have the opportunity to begin a binary aligned, and due to slight differences in precession rate, open up a large inclination difference between the component spin axes. Thus, for the spins to become misaligned with one another, we require

$$
\left|\frac{1}{T_{\text {precess }, 1}}-\frac{1}{T_{\text {precess }, 2}}\right|=\frac{1}{\text { system age }} .
$$

However, if the two spin precession periods are different but much shorter than the orbit precession period (Equation (1)), 
then the spins will just track the orbit orientation and will not become misaligned from either the orbit or each other. Therefore, we also require

$$
T_{\mathrm{sec}} \lesssim \max \left(T_{\text {precess }, 1}, T_{\text {precess }, 2}\right) .
$$

In our sample, we have two binaries with similar spectral types (GJ 569Bab and HD 130948BC) that are part of known triple systems. We have therefore produced a numerical demonstration of this effect for HD 130948BC, which has component $v \sin i$ values that differ, despite similar luminosities and spectral type. Although the mass ratio of this system has not been directly measured, we can follow the method of Dupuy et al. (2009b) and use a system age estimate and the component bolometric luminosities to estimate individual masses. This method predicts a slight mass difference due to the factor of $\sim 1.1$ difference in the luminosities. While the components should have very similar radii now, this was not the case when they were younger if they do indeed have slightly different masses. Because the radius is such an important factor in Equation (2), we assume for the purposes of this demonstration the average radii for these objects over the approximate age of the system ( $~ 500$ Myr; Dupuy et al. 2009b): $\left(R_{1}, R_{2}\right)=(0.158,0.150) R_{\odot}$ (Chabrier et al. 2000, DUSTY models), and corresponding masses $\left(M_{1}, M_{2}\right)=(0.06,0.05) M_{\odot}$ (Konopacky et al. 2010; Dupuy et al. 2009b). For the sake of this argument, we set $P_{1}=1.92 \mathrm{hr}$ and $P_{2}=1.82 \mathrm{hr}$ (i.e., $v_{\mathrm{eq}}=100 \mathrm{~km} \mathrm{~s}^{-1}$ ), so that the observed difference in $v \sin i$ will be due to inclination differences rather than rotation rate differences. Last, we chose apsidal motion constants $k_{2,1}=k_{2,2}=0.175$ and normalized moment of inertia $C_{1}=C_{2}=0.108$ (Leconte et al. 2011). We integrated the binary orbital parameters (starting with the observed $a=2.19$ AU and $e=0.16$; Konopacky et al. 2010) in the gravitational potential of the third star $\left(m_{3}=1.1 \mathrm{M}_{\odot}\right.$, $a=50 \mathrm{AU}, e=0.7 \mathrm{in}$ an orientation $\left.i=80^{\circ}, \omega_{\mathrm{in}}=45^{\circ}\right)$, using a secular code that uses the quadrupole approximation for the dynamics of the three stars (Fabrycky \& Tremaine 2007; Eggleton $\&$ Kiseleva-Eggleton 2001). In Figure 8, we show the results. On the short period $T_{\mathrm{sec}} \approx 10^{4} \mathrm{yr}$, the inner orbit precesses and goes through eccentricity oscillations, i.e., Kozai (1962) cycles. On the longer period $T_{\text {precess }} \approx 4 \times 10^{8} \mathrm{yr}$, the spins precess, and on an even longer period, $1 /\left(1 / T_{\text {precess, } 1}-1 / \tau_{\text {precess, } 2}\right) \approx 1 \times 10^{9} \mathrm{yr}$, the precession rates mix in phase (one gains by $\pi$, due to the slightly different masses and radii). The system is likely as young as 500-700 Myr (Dupuy et al. 2009b; Mullan \& MacDonald 2010). Therefore, it is plausible that the differential precession only recently opened a measurable angle between the rotation axes of these two components. In any case, this exercise suggests that no matter the true masses and radii of the components, Kozai cycles are quite possibly occurring in this system, and the differences in component $v$ sin $i$ 's are possibly just one manifestation of the precession of the inner binary. The rapidity of the oscillation period implies that the precession of the inner orbit and/or its eccentricity oscillation may be measurable; e.g., the eccentricity currently has a precision of $\sim 0.01$ (Dupuy et al. 2009b; Konopacky et al. 2010) and this numerical integration shows eccentricity changes of 0.01 per decade.

We have also performed an analogous simulation for the GJ 569Bab system, which exhibits $v$ sin $i$ differences of only $13 \mathrm{~km} \mathrm{~s}^{-1}$ as opposed to the $24 \mathrm{~km} \mathrm{~s}^{-1}$ difference in HD 130948BC. Using the orbital parameters of GJ 569Bab, which has a tighter separation $(\sim 1 \mathrm{AU})$, we find that the precession period for the system is much more rapid, on the order of 0.1 Myr. It is therefore substantially easier to have precession
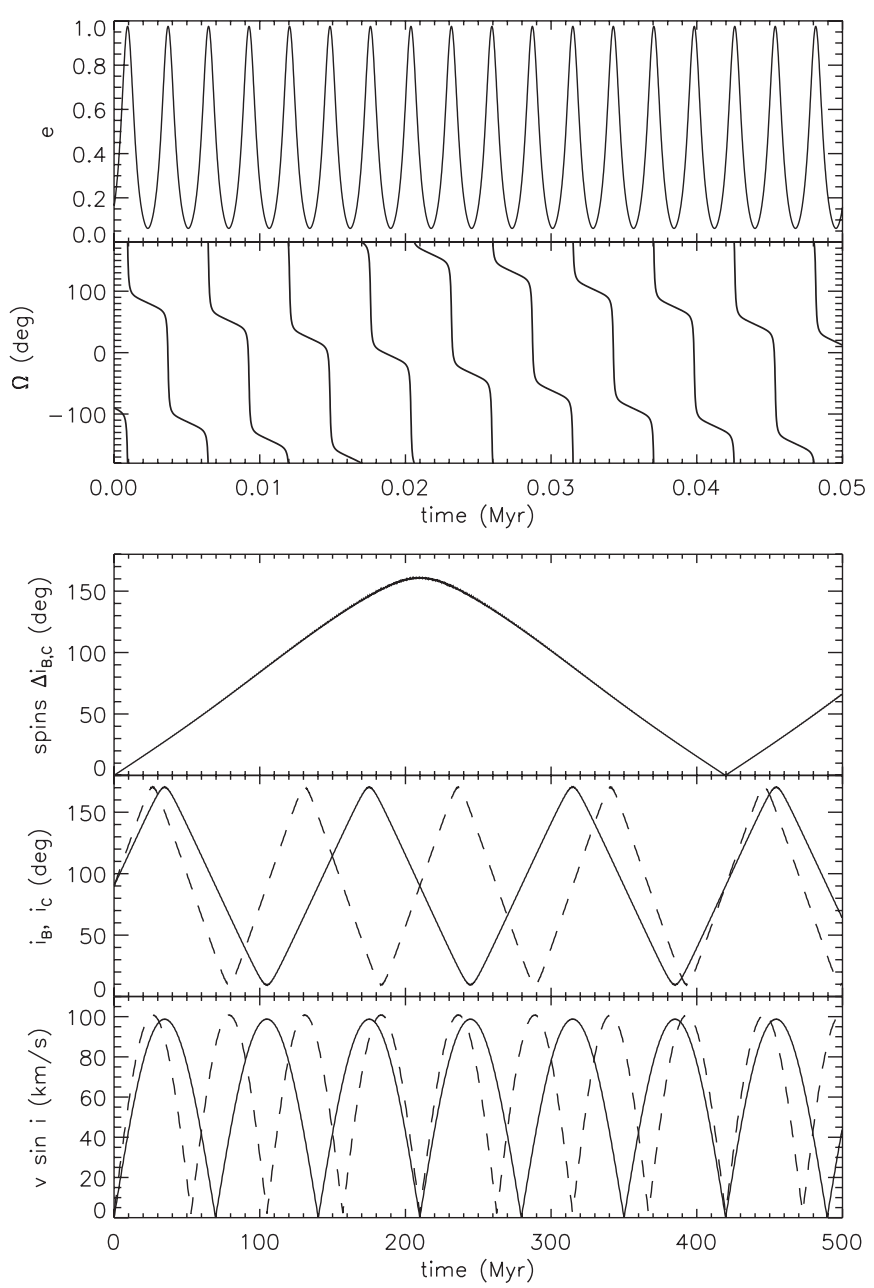

Figure 8. Top two panels: eccentricity oscillations and regression of the ascending node (in the reference frame of the A-BC orbit) for HD 130948BC due to the presence of HD 130948A. Based on the observed and modeled properties of the system, it could be undergoing Kozai (1962) oscillations on a $\sim 10^{4} \mathrm{yr}$ period. Bottom three panels: evolution of the angular difference in the spin vectors $\left(\Delta i_{\mathrm{B}, \mathrm{C}}\right)$, rotation axis inclination to the line of sight, and a possible resulting time history of $v \sin i$ of HD 130948B (solid line) and C (dashed line) due to the presence of HD 130948A. This effect could explain our $v \sin i$ measurements and do so within the expected lifetime of the system ( $500 \mathrm{Myr}$.)

occur for this system, making it plausible that secular perturbations are responsible for the $v \sin i$ difference in this system as well.

Three of the five systems with differing $v$ sin $i$ 's are not part of known triple systems. Allen et al. (2007) performed deep imaging around 2MASS $0746+20 \mathrm{AB}$ and 2 MASS $2140+16 \mathrm{AB}$ and found no comoving companions between 40 and $1000 \mathrm{AU}$ down to a mass limit of $\sim 0.05 M_{\odot}$. Although we are unaware of similar deep imaging for LP 349-25AB, current all-sky surveys do not reveal any bright sources within 1000 AU. This does not, however, rule out the possibility that these sources were previously members of higher order multiple systems, or had an interaction with an unrelated object. We also note that to our knowledge, none of the sources in our sample with consistent $v \sin i$ have additional companions.

\subsection{Implications for Radio Observations}

Two of our targets (2MASS 0746+20AB and LP 349-25AB) are known radio sources (Antonova et al. 2007; Phan-Bao et al. 2007). The components of these binaries exhibit rapid rotation $\left(\gtrsim 19 \mathrm{~km} \mathrm{~s}^{-1}\right)$. Both systems also have components 
with different $v \sin i$. This has interesting implications for determining which of the binary components is emitting in the radio, as the radio measurements are typically unresolved. For instance, Berger et al. (2009) used a $v$ sin $i$ for 2MASS $0746+20 \mathrm{AB}$ from unresolved measurements to derive a radius for the radio-emitting component, which they assumed to be the primary. Using the spatially resolved measurements of $v \sin i$ for these objects rather than an unresolved value (which at $27 \mathrm{~km} \mathrm{~s}^{-1}$ is nearly exactly the average of the spatially resolved values of 19 and $33 \mathrm{~km} \mathrm{~s}^{-1}$ that we obtain here) gives slightly different values for the radius. We can now derive the predicted radius for each component without assuming which is the emitting source and determine which gives a more plausible result. If we make the same assumption as Berger et al. (2009) that the rotation axis is perpendicular to the orbital plane, which was updated by Konopacky et al. (2010) to have an inclination of $138^{\circ} .2 \pm 0.5$, we derive velocities of $29 \pm 3 \mathrm{~km} \mathrm{~s}^{-1}$ and $50 \pm$ $3 \mathrm{~km} \mathrm{~s}^{-1}$ for the primary and secondary, respectively. Using the rotational period from Berger et al. (2009) of $124.32 \pm 0.11$ minutes gives a radius of $0.050 \pm 0.005 R_{\odot}$ for the primary and $0.085 \pm 0.005 R_{\odot}$ for the secondary. Assuming the total system mass and preliminary estimates of the component mass from Konopacky et al. (2010) that the mass of the primary is between 0.08 and $0.1 M_{\odot}$ while the secondary is between 0.05 and $0.07 M_{\odot}$, evolutionary models predict radii of $0.103-0.125 R_{\odot}$ for the primary and $0.094-0.096 R_{\odot}$ for the secondary. The implied discrepancy with the models is therefore $10 \sigma$ for the primary and $1.8 \sigma$ for the secondary. Given this, in conjunction with the unphysically small radius predicted for the primary, we postulate that the secondary is the source of the radio emission, which may be related to its more rapid rotation. Although the radius of $0.085 \pm 0.005 R_{\odot}$ is consistent to within the uncertainties of the value from Berger et al. (2009), the resulting overprediction of the radius by evolutionary models becomes slightly less severe because if the emission is from the secondary, the models predict that it will have a smaller radius. In either case, this analysis highlights the importance of obtaining fundamental parameters of binary components individually rather than from unresolved measurements. In addition, the results from our sample overall suggest that the assumption of rotational axis alignment with the orbital plane should be treated with much greater caution.

We can also speculate that the secondary component of LP $349-25 \mathrm{AB}$ is likely the radio source in the system, since it is rotating extremely rapidly. However, it is not improbable that both components are radio sources. Future Very Long Baseline Interferometry observations will probe the true origin of the radio emission in both of these system (G. Hallinan et al., in preparation). Furthermore, our results provide a guideline by which sources previously unobserved in the radio should be targeted. Binaries that have at least one component with $v \sin i>30 \mathrm{~km} \mathrm{~s}^{-1}$ would make excellent candidates for observation.

\section{SUMMARY}

Using the combination of high spatial and high spectral resolution afforded by the Keck II LGS AO system, we have measured component $v$ sin $i$ 's for a sample of 11 VLM binaries. Among the 22 objects measured, $80 \%$ are rapid rotators ( $v \sin i \gtrsim 10 \mathrm{~km} \mathrm{~s}^{-1}$ ), consistent with previous measurements for VLM objects. We found that five of the binaries surveyed had components with $v$ sin $i$ 's that differed by $>3 \sigma$. We explored potential causes for these differences, which must stem either from intrinsic velocity differences or from mutually inclined rotation axes, or a combination of both. Our analysis shows that perhaps both explanations are required to explain these five binaries, with two (2MASS $0746+20 \mathrm{AB}$ and $2 \mathrm{MASS} 2140+16 \mathrm{AB}$ ) having explainable intrinsic differences due to differing spectral types, while the other three (GJ569Bab, HD 130948BC, and LP 349-25AB) likely require a different explanation. We looked at the binary HD 130948BC as an example of a case in which secular torques are most likely causing the orbit and component spin vectors to evolve. This binary, in which Kozai oscillations are possibly at work, is an example of the impact of dynamical evolution on VLM binaries. One other binary in our sample with differing component velocities, GJ 569Bab, is part of a triple system and a similar analysis shows it too may be displaying the impact of secular torques. For LP 349-25AB, which is not part of a known triple system and spectral type differences do not seem to account for its vastly different $v \sin i$ 's, the explanation may rest with past dynamical encounters or perturbations by higher mass objects. Our results also have implications for the previous measurements of radioactivity in this system and $2 \mathrm{MASS} 0746+20 \mathrm{AB}$. We suggest that the secondary components are more likely the radio-emitting sources in these systems. This stresses the importance of measuring fundamental parameters of binary components individually rather than bootstrapping from unresolved measurements.

Continued monitoring of these systems will improve the precision to which parameters such as component mass and radius are measured, allowing for the correlation of the properties with rotational velocity. This will provide a new handle on the ages of these objects. Further, the objects that we found to be rapid rotators that have not yet been surveyed for radio emission are ideal targets for observation.

The authors thank the observing assistants Joel Aycock, Heather Hershley, Carolyn Parker, Gary Puniwai, Chuck Sorenson, Terry Stickel, and Cynthia Wilburn, and support astronomers Randy Campbell, Al Conrad, Marc Kassis, Jim Lyke, and Hien Tran for their help in obtaining the observations. We thank John Bailey for helpful advice regarding the analysis. We also thank an anonymous referee for helpful suggestions for the improvement of this document. This work was performed under the auspices of the U.S. Department of Energy by Lawrence Livermore National Laboratory under the Contract DE-AC52-07NA27344. Support for this work was provided by the NASA Origins Program (NNX1 OAH39G) and the NSF Science \& Technology Center for AO, managed by UCSC (AST-9876783). Some of the spectral analysis tools used in this study were developed in part by funding provided by the NSF/AAG Grant \#0908018. This publication makes use of data products from the Two Micron All Sky Survey, which is a joint project of the University of Massachusetts and the Infrared Processing and Analysis Center/California Institute of Technology, funded by the National Aeronautics and Space Administration and the National Science Foundation. The W.M. Keck Observatory is operated as a scientific partnership among the California Institute of Technology, the University of California and the National Aeronautics and Space Administration. The Observatory was made possible by the generous financial support of the W.M. Keck Foundation. The authors also wish to recognize and acknowledge the very significant cultural role and reverence that the summit of Mauna Kea has always had within the indigenous Hawaiian community. We are most fortunate to have the opportunity to conduct observations from this mountain. 


\section{REFERENCES}

Albrecht, S., Reffert, S., Snellen, I. A. G., \& Winn, J. N. 2009, Nature, 461, 373

Albrecht, S., Winn, J. N., Carter, J. A., Snellen, I. A. G., \& de Mooij, E. J. W. 2011, ApJ, 726, 68

Allen, P. R., Koerner, D. W., McElwain, M. W., Cruz, K. L., \& Reid, I. N. 2007, AJ, 133, 971

Antonova, A., Doyle, J. G., Hallinan, G., Golden, A., \& Koen, C. 2007, A\&A, 472,257

Artymowicz, P. 1983, Acta Astron., 33, 223

Bailer-Jones, C. A. L. 2004, A\&A, 419, 703

Bailey, J. I., III, White, R. J., Blake, C. H., et al. 2012, ApJ, 749, 16

Barnes, J. W., \& Fortney, J. J. 2003, ApJ, 588, 545

Bate, M. R. 1997, MNRAS, 285, 16

Bate, M. R., Lodato, G., \& Pringle, J. E. 2010, MNRAS, 401, 1505

Berger, E. 2006, ApJ, 648, 629

Berger, E., Rutledge, R. E., Phan-Bao, N., et al. 2009, ApJ, 695, 310

Blake, C. H., Charbonneau, D., \& White, R. J. 2010, ApJ, 723, 684

Blake, C. H., Charbonneau, D., White, R. J., Marley, M. S., \& Saumon, D. 2007, ApJ, 666, 1198

Bonnell, I., Martel, H., Bastien, P., Arcoragi, J.-P., \& Benz, W. 1991, ApJ, 377, 553

Bouvier, J., Rigaut, F., \& Nadeau, D. 1997, A\&A, 323, 139

Browning, M. K. 2008, ApJ, 676, 1262

Chabrier, G., Baraffe, I., Allard, F., \& Hauschildt, P. 2000, ApJ, 542, 464

Cieza, L. A., Padgett, D. L., Allen, L. E., et al. 2009, ApJ, 696, L84

Close, L. M., Siegler, N., Freed, M., \& Biller, B. 2003, ApJ, 587, 407

Delfosse, X., Forveille, T., Perrier, C., \& Mayor, M. 1998, A\&A, 331, 581

Donati, J.-F., Forveille, T., Collier Cameron, A., et al. 2006, Science, 311, 633

Duchêne, G. 2010, ApJ, 709, L114

Dupuy, T. J., Liu, M. C., \& Bowler, B. P. 2009a, ApJ, 706, 328

Dupuy, T. J., Liu, M. C., Bowler, B. P., et al. 2010, ApJ, 721, 1725

Dupuy, T. J., Liu, M. C., \& Ireland, M. J. 2009b, ApJ, 692, 729

Dupuy, T. J., Liu, M. C., \& Ireland, M. J. 2009c, ApJ, 699, 168

Edwards, S., Strom, S. E., Hartigan, P., et al. 1993, AJ, 106, 372

Eggleton, P. P., \& Kiseleva-Eggleton, L. 2001, ApJ, 562, 1012

Fabrycky, D., \& Tremaine, S. 2007, ApJ, 669, 1298

Forveille, T., Beuzit, J.-L., Delorme, P., et al. 2005, A\&A, 435, L5

Freed, M., Close, L. M., \& Siegler, N. 2003, ApJ, 584, 453

Gómez Maqueo Chew, Y., Stassun, K. G., Prša, A., \& Mathieu, R. D. 2009, ApJ, 699, 1196

Hale, A. 1994, AJ, 107, 306

Hallinan, G., Antonova, A., Doyle, J. G., et al. 2006, ApJ, 653, 690

Hallinan, G., Antonova, A., Doyle, J. G., et al. 2008, ApJ, 684, 644

Hauschildt, P. H., Allard, F., \& Baron, E. 1999, ApJ, 512, 377

Irwin, J., Aigrain, S., Bouvier, J., et al. 2009, MNRAS, 392, 1456

Jensen, E. L. N., Mathieu, R. D., Donar, A. X., \& Dullighan, A. 2004, ApJ, 600, 789

Johnson, J. A., \& Apps, K. 2009, ApJ, 699, 933

Jones, B. F., Fischer, D. A., \& Stauffer, J. R. 1996, AJ, 112, 1562

Kiseleva, L. G., Eggleton, P. P., \& Mikkola, S. 1998, MNRAS, 300, 292

Konopacky, Q. M., Ghez, A. M., Barman, T. S., et al. 2010, ApJ, 711, 1087
Kozai, Y. 1962, AJ, 67, 591

Kundurthy, P., Meyer, M. R., Robberto, M., Beckwith, S. V. W., \& Herbst, T. 2006, AJ, 132, 2469

Lai, D., Foucart, F., \& Lin, D. N. C. 2011, MNRAS, 412, 2790

Leconte, J., Lai, D., \& Chabrier, G. 2011, A\&A, 528, A41

Livingston, W., \& Wallace, L. 1991, An Atlas of the Solar Spectrum in the Infrared from 1850 to $9000 \mathrm{~cm}^{-1}$ (1.1 to 5.4 micrometer) (Tucson, AZ: National Solar Observatory)

Martín, E. L., Koresko, C. D., Kulkarni, S. R., Lane, B. F., \& Wizinowich, P. L. 2000, ApJ, 529, L37

McCabe, C., Ghez, A. M., Prato, L., et al. 2006, ApJ, 636, 932

McLean, I. S., Graham, J. R., Becklin, E. E., et al. 2000, Proc. SPIE, 4008, 1048

Mohanty, S., \& Basri, G. 2003, ApJ, 583, 451

Monin, J., Ménard, F., \& Peretto, N. 2006, A\&A, 446, 201

Monnier, J. D., Zhao, M., Pedretti, E., et al. 2007, Science, 317, 342

Mullan, D. J., \& MacDonald, J. 2010, ApJ, 713, 1249

Nguyen, D. C., Jayawardhana, R., van Kerkwijk, M. H., et al. 2009, ApJ, 695, 1648

Osten, R. A., Hawley, S. L., Bastian, T. S., \& Reid, I. N. 2006, ApJ, 637, 518

Patience, J., Ghez, A. M., Reid, I. N., \& Matthews, K. 2002, AJ, 123, 1570

Phan-Bao, N., Osten, R. A., Lim, J., Martín, E. L., \& Ho, P. T. P. 2007, ApJ, 658,553

Potter, D., Martín, E. L., Cushing, M. C., et al. 2002, ApJ, 567, L133

Reid, I. N., Gizis, J. E., Kirkpatrick, J. D., \& Koerner, D. W. 2001, AJ, 121, 489

Reiners, A., \& Basri, G. 2007, ApJ, 656, 1121

Reiners, A., \& Basri, G. 2008, ApJ, 684, 1390

Reiners, A., \& Basri, G. 2010, ApJ, 710, 924

Reiners, A., Seifahrt, A., Käufl, H. U., Siebenmorgen, R., \& Smette, A. 2007, A\&A, 471, L5

Rojas-Ayala, B., Covey, K. R., Muirhead, P. S., \& Lloyd, J. P. 2010, ApJ, 720, L113

Schlaufman, K. C., \& Laughlin, G. 2010, A\&A, 519, A105

Scholz, A., Eislöffel, J., \& Mundt, R. 2009, MNRAS, 400, 1548

Scholz, A., Irwin, J., Bouvier, J., et al. 2011, MNRAS, 413, 2595

Sengupta, S., \& Marley, M. S. 2010, ApJ, 722, L142

Shu, F. H., Tremaine, S., Adams, F. C., \& Ruden, S. P. 1990, ApJ, 358, 495

Siegler, N., Close, L. M., Cruz, K. L., Martín, E. L., \& Reid, I. N. 2005, ApJ, 621,1023

Siegler, N., Close, L. M., Mamajek, E. E., \& Freed, M. 2003, ApJ, 598, 1265

Simon, M., Bender, C., \& Prato, L. 2006, ApJ, 644, 1183

Stassun, K. G., Mathieu, R. D., Mazeh, T., \& Vrba, F. J. 1999, AJ, 117, 2941

Strom, S. E., Edwards, S., \& Skrutskie, M. F. 1993, Protostars and Planets III (Tucson, AZ: Univ. of Arizona Press), 837

Terndrup, D. M., Stauffer, J. R., Pinsonneault, M. H., et al. 2000, AJ, 119, 1303

Triaud, A. H. M. J., Collier Cameron, A., Queloz, D., et al. 2010, A\&A, 524, A25

Winn, J. N., Fabrycky, D., Albrecht, S., \& Johnson, J. A. 2010, ApJ, 718, L145

Winn, J. N., Johnson, J. A., Albrecht, S., et al. 2009, ApJ, 703, L99

Wizinowich, P. L., Le Mignant, D., Bouchez, A. H., et al. 2006, PASP, 118, 297

Zapatero Osorio, M. R., Lane, B. F., Pavlenko, Y., et al. 2004, ApJ, 615, 958

Zapatero Osorio, M. R., Martín, E. L., Bouy, H., et al. 2006, ApJ, 647, 1405 